OPEN ACCESS

Edited by:

Javier Echeverria,

University of Santiago, Chile

Reviewed by:

Mattan Levi,

Tel Aviv University, Israel

Parveen Bansal,

Baba Farid University of Health

Sciences, India

*Correspondence:

Narendra Bhatt

drnsbhatt@gmail.com

Specialty section:

This article was submitted to

Ethnopharmacology,

a section of the journal

Frontiers in Pharmacology

Received: 15 November 2020

Accepted: 11 May 2021

Published: 03 June 2021

Citation:

Bhatt N and Deshpande M (2021) A

Critical Review and Scientific

Prospective on Contraceptive Therapeutics from Ayurveda and Allied

Ancient Knowledge.

Front. Pharmacol. 12:629591.

doi: 10.3389/fphar.2021.629591

\section{A Critical Review and Scientific Prospective on Contraceptive Therapeutics from Ayurveda and Allied Ancient Knowledge}

\author{
Narendra Bhatt ${ }^{1 *}$ and Manasi Deshpande ${ }^{2}$ \\ ${ }^{1}$ CRIA Consultant Pvt. Ltd. Mumbai, Mumbai, India, ${ }^{2}$ Department of DravyagunaVigynan, Bharati Vidyapeeth Deemed to be \\ University, College of Ayurved, Pune, India
}

Commonly used synthetic or prescribed hormonal drugs are known to interfere with the endocrine system and may have adverse reproductive, neurological, developmental, and metabolic effects in the body. These may also produce adverse effects such as polycystic ovarian disorder, endometriosis, early puberty, infertility or toxicity to gonads, testicular germ cell cancer, breast or prostate cancer, brain developmental problems, and even birth defects. Globally, the emergence of renewed interest in natural products for reproductive health is on the rise, which offers opportunities for new contraceptive developments. The search for alternate, safer contraceptive products or agents of natural origin is of scientific interest. Ayurvedic classical texts offer knowledge and information about the reproductive function and therapeutics including those for enhancement and limiting male and female fertility. Review of ancient, medieval, and recent-including texts on erotica that provide information on approaches and large numbers of formulations and drugs of plant, mineral or animal origin-claimed to have sterilizing, contraceptive, abortifacient, and related properties is presented. Few among these are known to be toxic and few are not so common. However, most of the formulations, ingredients, or modes of administration have remained unattended to, due to issues related to consumer compliance and limitations of standardization and lack of appropriate validation modalities. Several of these ingredients have been studied for their phytoconstituents and for the variety of pharmacological activities. Efforts to standardize several classical dosage forms and attempts to adapt to modern technologies have been made. List of formulations, ingredients, and their properties linked with known constituents, pharmacological, biological, and toxicity studies have been provided in a series of tables. The possible effectiveness and safety of selected formulations and ingredients have been examined. Suggestions based on new drug delivery systems integrated with advances in biotechnology, to provide prospects for new therapeutics for contraception, have been considered. Ayurveda is built on a holistic paradigm of biological entity rather than limited gonadal functions. Graphic presentation of a few carefully chosen possibilities has been depicted. New approaches to standardization and ethnopharmacological validation of natural contraceptive therapeutics may offer novel mechanisms and modalities and therapeutic opportunities to satisfy unmet needs of contraception.

Keywords: natural contraceptive, herbal contraceptive, ayurved contraceptive, reproductive health and traditional medicine, contraceptive traditions 


\section{INTRODUCTION}

The world population is expected to reach more than 11 billion by 2050 (Census of India, 2011). Population in the world is currently (2020) growing at a rate of around $1.05 \%$ per year. The current average population increase is estimated at 81 million people per year and current world population is 7.9 billion as of March 2021 (World Population Clock, 2021). This burgeoning population particularly in developing countries is a matter of concern for social, economic, and environmental reasons in terms of providing food, shelter, and life. The challenge of dealing with an ever-increasing population has been dealt with largely by conventional medicine using different methods of contraception such as oral contraceptive pills, intrauterine contraceptive devices, and barrier devices. These devices, techniques, and drugs seem to have been efficiently practiced for contraception but with many reported adverse effects as well as failure resulting in unwanted pregnancy. (Dutta, 2013).

\section{BIRTH CONTROL HISTORY}

Technically, birth control can be defined as the methods, procedures, or practices that are implemented to prevent conception leading to pregnancy in women. The term can be associated with contraception and family planning where knowledge about birth control is equally important.

The Egyptian Ebers Papyrus from 1550 BCE and the Kahun Papyrus from 1850 BCE have some of the earliest documented descriptions of birth control: the use of honey, acacia leaves, and lint to be placed in the vagina to block sperm. (Lipsey et al., 2005; Cuomo, 2010).
In medieval Europe, any effort to halt pregnancy was deemed immoral by the Catholic Church, (Cuomo, 2010), although it is believed that women of the time still used a number of birth control measures such as coitus interrupts and inserting lily root and rue into the vagina. Women in the middle ages were also persuaded to tie weasel-a small wild animal-testicles around their thighs during sex to prevent pregnancy. The oldest condoms discovered to date were recovered in the ruins of Dudley Castle in England and date back to 1640 . They were made of animal gut and were most likely used to prevent the spread of sexually transmitted diseases during the English Civil War (Jon, 2012). Casanova, living in 18th-century Italy, described the use of a lambskin covering to prevent pregnancy; however, condoms only became widely available in the 20th century (Cuomo, 2010).

\section{Modern Methods to Control Fertility (World Health Organization, 2020)}

Several methods currently used to curb for contraception are presented (Figure 1).

\section{ADVERSE EFFECTS}

Commonly used synthetic or prescribed hormonal drugs are known to interfere with the endocrine system and may have adverse reproductive, neurological, developmental, and metabolic effects in the body. These may cause polycystic ovarian disorder, endometriosis, early puberty, infertility, toxicity to gonads, testicular germ cell cancer, breast or prostate cancer, brain developmental problems, and even birth defects. The search for alternate and safer means/drugs to prevent

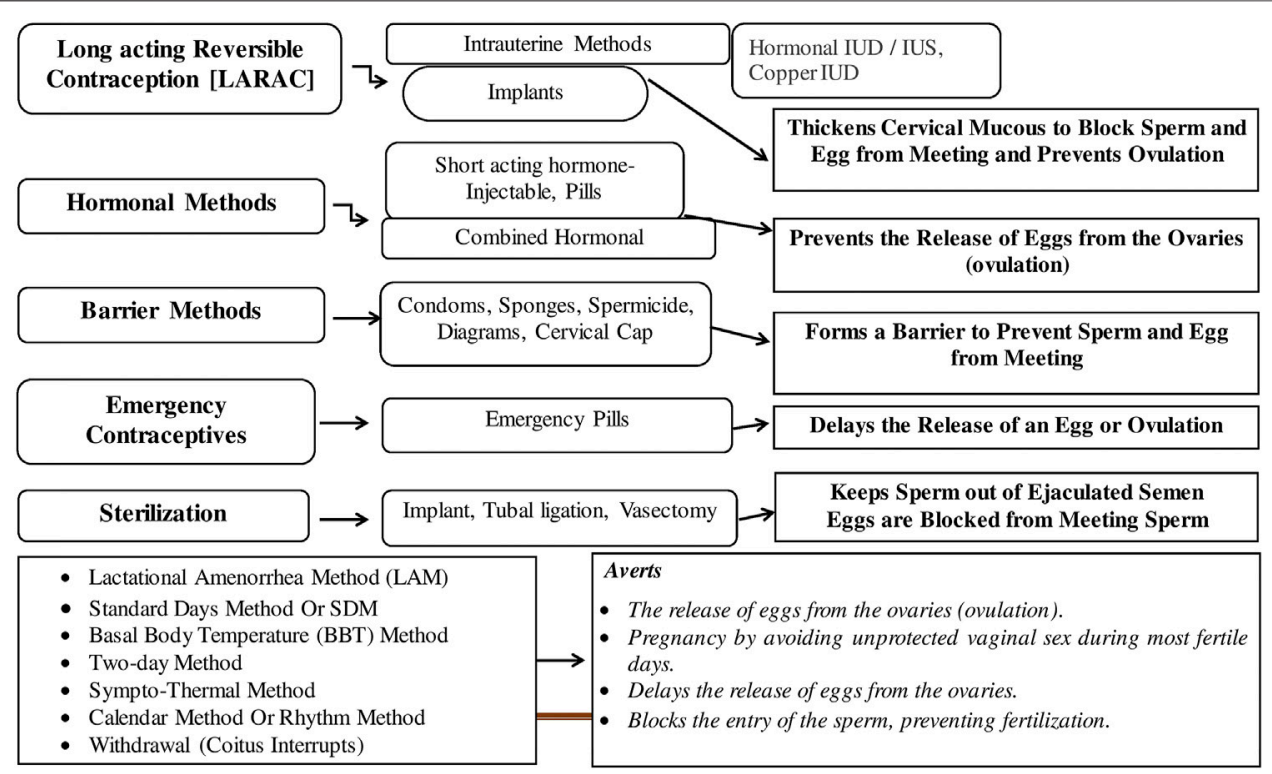

FIGURE 1 | Modern methods of birth control. 
birth is an open-ended area of scientific research. It is always an appealing idea to further research to develop contraceptive drugs of natural origin that have high efficacy without any adverse effects on the reproductive system.

\section{UNMET NEEDS}

According to a recent report from the Guttmacher Institute, 214 million women of reproductive age in the developing world who want to avoid pregnancy are not using a modern contraceptive method. These women are considered to have an "unmet need" for modern contraception, with 59 million relying on traditional methods such as abstinence and withdrawal and 155 million simply using no contraception at all. (Elizabeth et al., 2020).

India's total fertility rate (TFR) may have declined significantly over the years, but there remain significant challenges in family planning according to new research. In an Economic and Political Weekly article, Purushottam M. Kulkarni of Jawaharlal Nehru University suggested that there is a significant unmet need for contraception in India. Data from National Family Health Surveys (NFHS) have shown that while there was a decline in the unmet need for contraceptive services from 1992-93 (NFHS1) through to 2005-06 (NFHS-3), and between 2005-06 and 201516 (NFHS-4), there has not been any significant improvement in access to contraception. (Mint, 2020).

\section{SIGNIFICANCE OF REVIEW}

Despite obvious success, the rise in population continues to remain a medical challenge due to reasons of social, economic, personal, and biological consequences. Though well-established contraceptive drugs and measures have been utilized, the long term and excessive use of hormonal contraceptives are of serious concerns due to their probable adverse effects. There is need to explore the alternative or new possibilities.

The search for an effective and safe contraceptive agent remains a challenge. Contraceptive drugs of natural origin are of all-time research interests. Traditional systems of medicine like Ayurveda address all issues related to health and illnesses based on the principle of equilibrium between the biosphere and cosmosphere, which include reproductive phenomenon. Ayurvedic pharmacopoeia has formulations and ingredients that are attributed to affect coitus, spermatogenesis, and ovulation, uterine, fetal, and placental activities. These include emmenagogues, ecbolic drugs, contraceptives, uterine sedatives for females, and depurate or drugs that hamper male sexual and reproductive capabilities, affect fluidity or motility of the seminal fluid, destroy sperms, or impede libido.

A large number of drugs are known to have sterilizing, contraceptive, and abortifacients properties. However, these indigenous means and drugs were extensively used even in rural or tribal cultures until the 20th century, when there has been no noteworthy systematic or scientific efforts to study these aspects except for a few intermittent studies. While the list of such ingredients is quite big, unusually small scientific data are available about the nature of their active components and about their mechanisms of action.

As biotechnology-based advances open up new vistas in biomedical research, it will be of interest to examine the subject of contraception once again, as in Ayurveda, in the light of present-day pharmacology for future possibilities.

A thoughtful attempt has been made here to explore Ayurvedic and scientific aspects of formulations and ingredients as described in multiplicity of classical texts covering different facets of contraception.

\section{METHODOLOGY}

Ancient classical texts, medieval compendia, and other pertinent texts were assessed for enlisting different methods used for contraception and to enlist formulations and ingredients used for a variety of activities that could be pharmacologically linked to contraception. Specific search was undertaken for any existing review that could add to information on the subject. A systematic review of published articles on the subjects related to contraception was undertaken. The description of methods used in the experimental animal models, and the antifertility effect of active ingredients, their doses, safety, and toxicity were examined. Ninety-four plants and six minerals are reported in this review having a variety of contraceptive activities.

Flowchart of the systematic review process to search for contraceptive plants is presented. (Figure 2).

\section{CONTRACEPTIVES IN AYURVEDA AND MEDIEVAL SANSKRIT LITERATURE}

Ayurvedic literature is rife with thousands of formulations and has about 1100 ingredients attributed with well-defined therapeutic approaches including reproduction. There are references to temporary or permanent sterilization. Search for contraception from traditional knowledge of Ayurveda has been of interest to the Central Drug Research Institute, Council of Scientific and Industrial Research under Ministry of Science and Technology, and the Central Council for Research in Ayurvedic Sciences under Ministry of Health (now Ministry of AYUSH), bodies under the Government of India. Several other private and industry organizations had undertaken studies in the past. However, there is a need to revive research interest in Ayurveda in reproductive biology for safe, low-cost, userfriendly, and reliable therapeutic solutions to satisfy different contraception requirements.

\section{Vedic Period (1500-500 BCE)}

Regulated sexual life or abstinence from sex was considered the ideal method of contraception in Vedic times. The emphasis was more on propensity of the right, healthy progeny. Indirect references to contraception can be found in the Atharva Veda.

The use of drugs leading to impotence as punishment meted out to a person committing social sins or to an enemy or infliction 


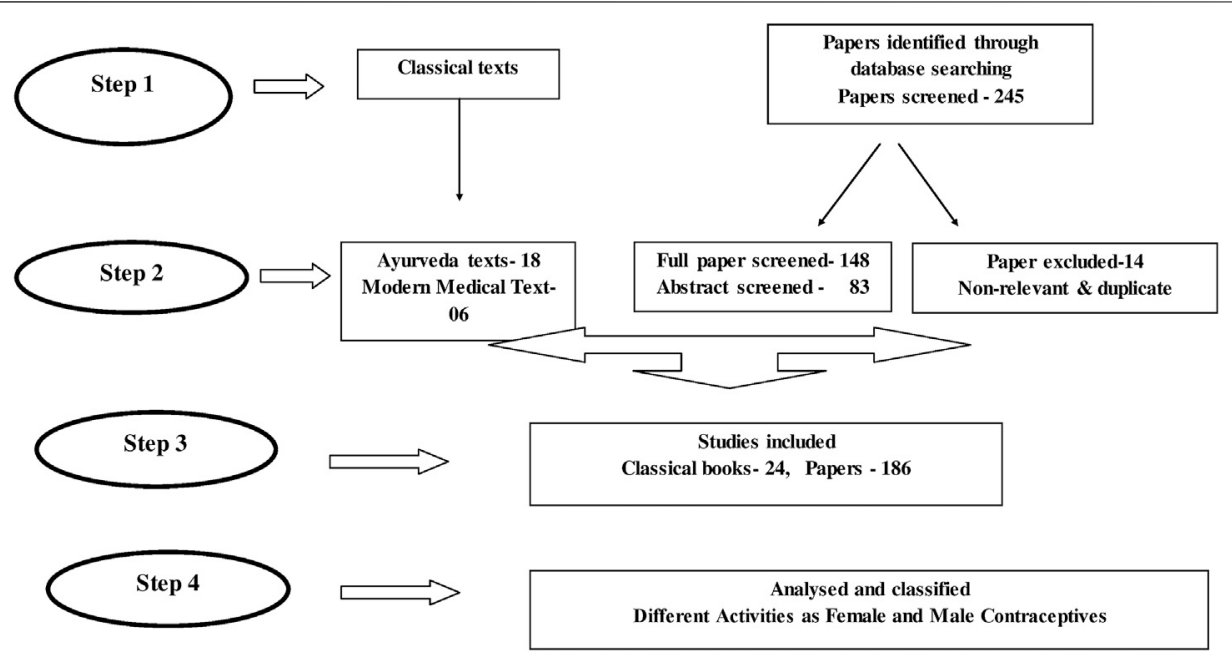

FIGURE 2 | Flowchart of the systemic review process searching for contraceptive plants.

of injury to two cords situated near the scrotum or the scrotum itself, to put an end to one's desire for progeny were in practice. These can be considered as references for use of drugs to prevent conception, vasectomy, and castration, respectively (Satvalekar, 1958a).

A mechanical device made of stone to obstruct multiple channels of Yoni-the vaginal cavity to prevent conception has been mentioned. This could be considered as the earliest form of an intrauterine contraceptive device. Similarly, artificially induced changes to make the vaginal cavity rough or dry, besides its mechanical obstruction for futile coitus have been mentioned (Satvalekar, 1958b). This reference reflects some chemical changes to be produced artificially, probably in the cervical mucus obstructing the entry of sperms, or in the endometrium influencing the implantation of the zygote and a mechanical barrier in the vaginal canal. (Tewari and Chaturvedi, 1981). In Brhadaranyaka Upanishad, a breath exercise is advised during coitus to avoid conception (Dash and Basu, 1968).

\section{Samhita Period: (300-500 BCE)}

Though Charak Samhita, Sushrut Samhita, and Ashtang Sangraha-Bruhatryee, the three ancient most Ayurveda treatises, have elaborated the subject of reproduction extensively, there are no direct references to contraception.

Kshetra-the female reproductive system as the field, $a m b u$-the nutrient fluids, bija-the sperm or ovum as the seed, rutukal-the ideal ovulatory period, marga-the female canal, Vayu-the neural system, and hrid-the psychological status are considered the essential factors for conception. Any or more of these factors if influenced artificially can lead to a failure of conception. The shukravaha srotas and aartavavaha srotas representing seminal and menstrual flows, respectively, are among the 13th intrinsic and interdependent biological pathways or channels (that could be explained based on now prevalent means of system biology). This early knowledge could pave the way for the development of different kinds of contraceptive methods prevailing in the present scenario, and all of them influence one or the other factors that have been explained in the ancient classics (Vagbhatt, 2000; Sushrut, 2002).

Contraceptive activities in the context of Ayurvedic principle of fertility are explained in Figure 3.

\section{Medieval Period (1000 AD to 1900 AD)}

Rajamartanda written in the 11th century is probably one of the earliest texts to mention a specific prescription for contraceptives. Compendia texts like Bhava Prakash, Yoga Ratnakara, Bhaishajya Ratnavali, Gadanigrah, and several others prescribe many herbal and herbo-mineral contraceptive preparations for local and oral use by men and women.

By the 11th century, the oriental connectivity that had sociocultural effects also brought in practices to prevent conception or induce abortion. References to oral and local contraceptives are found in Bruhad Yoga Tarangini and RatiRahasya [AD800], RasaPrakashSudhakar [AD1300], Panchasayaka, Smaradeepika and RasaRatnaSamuccchay [AD1400], RatiManjiri [AD1500], Kandarpchudamani [AD1577], AnnangaRang, Bhavprakash and YogaRatnakar [AD1600], YogaRatnaSamucchaya [AD 1800], and Brihan Nighantu Ratnakar and BhaishyyaRatnaval i[AD 1900].

The subject of contraceptives in ancient times dealt not only with medieval medicine but also with art and the literary works of poets, playwrights, and philosophers. Like Kama Sutra, the famous text on erotica, a large number of books in the 19th century contain various recipes for contraception and for inducing abortions and diverse birth control practices.

\section{Some of the most prescribed practices and recipes for preventing conception are as follows.}

1. Local Contraceptives for Females

Vaginal fumigation or application before coitus with (1) moistened Saindhava lavana (Rock salt) with Til (Sesame) oil. (Jugnu and Sharma, 2011), (2) wood of Neem (Azadirachta 


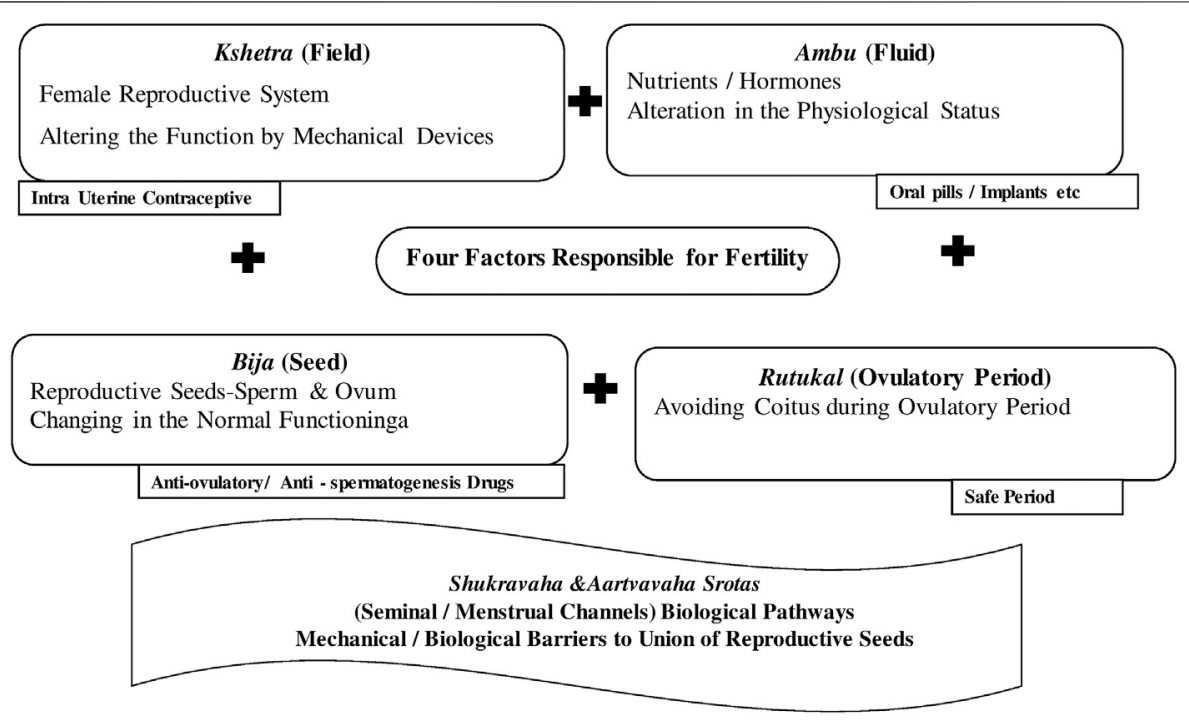

FIGURE 3 | Contraception in the context of Ayurvedic principle of fertility.

indica A. Juss. ) before coitus (Tripathi, 1969), and (3) powdered root of Dhattura (Datura metel L.) plucked on the 14 th $^{\circ}$ day (dark night) of the lunar month[Indradev, 1998] or tying the waist with roots (Lakmipatishashtri, 1983).

2. Oral Contraceptives

- Powder of Pippali (Piper longum L.) and Vidanga (Embelia ribes Burm.f.) with Tankana (Borax) taken in equal quantity in fertile phase with milk (Lakmipatishashtri, 1983).

- Flowers of Japa (Hibiscus rosa-sinensis L.): immediately after the delivery of a child (Bhavamisra, 1961; Lakmipatishashtri, 1983) or with Kanji (fermented drink) along with 48 grams of old jaggery to be taken for $3^{\circ}$ days in the fertile phase. (Lakmipatishashtri, 1983).

- Root of Tanduliyaka (Amaranthus spinosus L.) with Tandulodaka (rice water) to be taken after menstruation for $3^{\circ}$ days. (Lakmipatishashtri, 1983).

- Powders of Talisa patra (Abies spectabilis (D.Don) Mirb.) and Gairika (Red Ochre, $\mathrm{Fe}_{2} \mathrm{O}_{3}$ ) in equal parts to be consumed on the 4 th day of menstruation with water. (Lakmipatishashtri, 1983).

- Aqueous extract of Rasanjana (Extract of Berberis aristata DC.), Hemavati (Sweta - Vacha) (Iris $\times$ germanica L.), and Vayastha (Terminalia chebula Retz.) with cold water. (Rajeshwaradatta, 2001).

- Powders of Amla (Phyllanthus emblica L.), Arjuna (Terminalia arjuna (Roxb. ex DC)), and Abhaya (Terminalia chebula Retz.) with water. (Rajeshwaradatta, 2001).

- Paste made of the root of Chitraka (Plumbago zeylanica L.) mixed with Nirgundi (Vitex negundo L.) juice given orally in the dose of one $12^{\circ} \mathrm{gm}$ with honey. (Lakmipatishashtri, 1983).

- Powder of seeds of Sarshapa (Brassica rapa L.) with Tanduliyam (Amaranthus spinosus L.) and Sarkara
(Sugar candy) pounded with Tandulodaka (rice water) given with milk. (Jugnu and Sharma, 2011).

- Ashes of Sehund stem (Euphorbia neriifolia L.), $12^{\circ} \mathrm{g}$ daily. (Kuchimara, 2007).

- Rhizome of Haridra (Curcuma longa L.) daily during the $3^{\circ}$ days of menstruation followed by an additional $3^{\circ}$ days (Kuchimara, 2007).

- Powders of Krishna Jeeraka (Carum carvi L.), Karchooram (Hedychium spicatum Sm.), Nagakesara (Mesua ferrea L.), Haritaki (Terminalia chebula Retz.), Kalonji (Nigella sativa L.), and Kayaphala (Myrica nagi Thunb.) made into pills in the size of ziziphus fruit for $7^{\circ}$ days. (Kuchimara, 2007).

3. Abortifacient

- Root of Sweta Aparajita (Clitoria ternatea L.), Kakadani (Sarngesta)(Cardiospermum halicacabum L.) or Punarnava (Boerhavia diffusa L.) with oil of Eranda (Ricinus communis L.) - Patradanda (stem of leaf) to be inserted in the vagina (Rajamartanda), 1966; $\quad$ Tripathi, 1969; Lakmipatishashtri, 1983

- Devalaya Churna (scrapped lime powder from the wall of temple) $12^{\circ} \mathrm{g}$ with water. (Lakmipatishashtri, 1983; Indradev, 1998).

- Seeds of Grnjana (Carrot) (Daucus carota L) with roots of Tuvari (Cajanus cajan (L.) Huth) and Sindura (lead oxide).

- Ghotipurisa (feces of mare) mixed with Kanji, filtered, and mixed with rock salt, Ugra (Apium graveolens L.), and AsuriTaila (Oil of Brassica juncea (L.) Czern.) with Visha (Aconitum chasmanthum Stapf ex Holmes) (Lakmipatishashtri, 1983).

Plant and mineral drugs mentioned as contraceptives in the Ayurvedic classical texts are given in Table $\mathbf{1}$.

It is observed that 79 plant drugs and six mineral drugs are used as abortifacients, oral contraceptives, or as local applications 
TABLE 1 | List of plant and metal drugs as contraceptives in Ayurveda classics. Vertical column numbers indicate AaartavJanan - Emmenagogue (1),

Aparapatan - placental expulsion (2), Garbhanuloman/Garbhapatkar-Abortifacient or Garbhastravakar-expel Fetus (3), Garbhanirodhak Contraceptives (4),

Garbhashayasancochak-Ecbolic (5), Shandhyakar/Pumstvopadhatin - drugs that hamper male sexual or reproductive capability (6), and Shukrashodhan - Depurates (7).

\begin{tabular}{|c|c|c|c|c|c|c|c|c|c|}
\hline $\begin{array}{l}\text { Sr. } \\
\text { No. }\end{array}$ & $\begin{array}{l}\text { Sanskrit } \\
\text { name }\end{array}$ & $\begin{array}{l}\text { Botanical } \\
\text { name }\end{array}$ & (1) & (2) & (3) & (4) & (5) & (6) & (7) \\
\hline & Aguru & Aquilaria malaccensis Lam. & & $\sqrt{ }$ & & & & & \\
\hline & Ahiphen & Papaver somniferum L. & & & & & & $\sqrt{ }$ & \\
\hline & Amalaki & Phyllanthus emblica $L$ & & & & $\sqrt{ }$ & & & \\
\hline & Ashok & Saraca asoca (Roxb.) J.J.de Wilde & $\sqrt{ }$ & & & & $\sqrt{ }$ & & \\
\hline & Asuri & Brassica juncea (L.) Czern & & & $\sqrt{ }$ & & & & \\
\hline & Arjuna & Terminalia arjuna (Roxb. ex DC.) Wight \&Arn. & & & & $\sqrt{ }$ & & & \\
\hline & Bhanga & Cannabis sativa L. & & & & & & $\sqrt{ }$ & $\sqrt{ }$ \\
\hline & Bhurjapatra & Betula utilis D. Don & & $\sqrt{ }$ & $\sqrt{ }$ & & & & \\
\hline & Chandan & Santalum album L. & & & & & & $\sqrt{ }$ & \\
\hline & Chavya & Piper retrofractum Vahl & & $\sqrt{ }$ & & & & & \\
\hline & Chirbilva & Holoptelea integrifolia (Roxb.) Planch. & & & $\sqrt{ }$ & & & & \\
\hline & Chitraka & Plumbago zeylanica L. & $\sqrt{ }$ & $\sqrt{ }$ & $\sqrt{ }$ & $\sqrt{ }$ & & & \\
\hline & Chuka & Rumex acetosa L. & & & & & & $\sqrt{ }$ & \\
\hline & Devdaru & Cedrus deodara (Roxb. ex D. Don) G. Don & $\sqrt{ }$ & $\sqrt{ }$ & & & & & \\
\hline & Dhanyak & Coriandrum sativum L. & & & & & & $\sqrt{ }$ & \\
\hline & Dhattura & Datura metel L. & & & & $\sqrt{ }$ & & $\sqrt{ }$ & \\
\hline & Ela & Elettaria cardamomum (L.) Maton & & $\sqrt{ }$ & $\sqrt{ }$ & & & & \\
\hline & Eranda & Ricinus communis L. & & & $\sqrt{ }$ & & & & \\
\hline & Eshvari & Aristolochia indica L. & & & $\sqrt{ }$ & & $\sqrt{ }$ & & \\
\hline & Grnjana & Daucus carota L. & & & $\sqrt{ }$ & & & & \\
\hline & Haridra & Curcuma longa L. & & & & $\sqrt{ }$ & & & \\
\hline & Haritaki & Terminalia chebula Retz. & & & & $\sqrt{ }$ & & & \\
\hline & Harmal & Peganum harmala L. & & & & & $\sqrt{ }$ & & \\
\hline & Hemavati & Iris germanica L. & & & & $\sqrt{ }$ & & & \\
\hline & Hingu & Ferula assa-foetida $L$. & & & $\sqrt{ }$ & & & & \\
\hline & Hirabol & Commiphora myrrha (Nees) Engl. & & & & & $\sqrt{ }$ & & \\
\hline & Japa & Hibiscus rosa-sinensis L. & & & & $\sqrt{ }$ & & & \\
\hline & Karchuram & Hedychium spicatum Sm. & & & & $\sqrt{ }$ & & & \\
\hline & Kadamb & Neolamarckia cadamba (Roxb.) Bosser & & & & & & & $\sqrt{ }$ \\
\hline & Kakadani (Sarngesta) & Cardiospermum halicacabum L. & & & $\sqrt{ }$ & & & & \\
\hline & Kakamachi & Solanum nigrum L. & & & & & & $\sqrt{ }$ & \\
\hline & Karpas & Gossypium herbaceum L. & $\sqrt{ }$ & & & & $\sqrt{ }$ & & \\
\hline & Karpur & Cinnamomum camphora (L.) J. Presl & & & & & & $\sqrt{ }$ & \\
\hline & Kasani & Cichorium intybus $\mathrm{L}$. & & & & & & $\sqrt{ }$ & \\
\hline & Kayaphala & Myrica nagi Thunb. & & & & $\sqrt{ }$ & & & $\sqrt{ }$ \\
\hline & Ketaki & Pandanus tectorius Parkinson ex Du Roi & & & $\sqrt{ }$ & & & & \\
\hline & Krishna Jeeraka & Carum carvi L. & & & $\sqrt{ }$ & $\sqrt{ }$ & & & \\
\hline & Kulattha & Vigna unguiculata (L.) Walp. & $\sqrt{ }$ & $\sqrt{ }$ & & & & $\sqrt{ }$ & \\
\hline & Kushtha & Aucklandia costus Falc & $\sqrt{ }$ & $\sqrt{ }$ & $\sqrt{ }$ & & & & $\sqrt{ }$ \\
\hline & Langali & Gloriosa superba L. & & & $\sqrt{ }$ & & $\sqrt{ }$ & & \\
\hline & Lodhra & Symplocos racemosa Roxb. & & & & & $\sqrt{ }$ & & \\
\hline & Mandukparni & Centella asiatica (L.) Urb. & & $\sqrt{ }$ & & & & & \\
\hline & Mocharas & Bombax ceiba L. & & & & & $\sqrt{ }$ & & \\
\hline & Nagakesara & Mesua ferrea L. & & & & $\sqrt{ }$ & & & \\
\hline & Nagdamani & Artemisia nilagirica (C. B. Clarke) Pamp. & & & $\sqrt{ }$ & & & & \\
\hline & Neem & Azadirachta indica A. Juss. & & & & $\sqrt{ }$ & $\sqrt{ }$ & & \\
\hline & Nimbu & Citrus $\times$ aurantium L. & & & & & & $\sqrt{ }$ & \\
\hline & Nilophar & Nymphaea alba L. & & & & & & $\sqrt{ }$ & \\
\hline & Nirgundi & Vitex negundo L. & & & $\sqrt{ }$ & $\sqrt{ }$ & & $\sqrt{ }$ & \\
\hline & Pippali & Piper longum L. & & $\sqrt{ }$ & & $\sqrt{ }$ & & & \\
\hline & Punarnava & Boerhavia diffusa L & & & $\sqrt{ }$ & & & & \\
\hline & Rasanjana & Berberis aristata DC. & & & & $\sqrt{ }$ & & & \\
\hline & Rason & Allium cepa L. & & & & & & $\sqrt{ }$ & \\
\hline & Sarshapa & Brassica rapa L. & & & & & $\sqrt{ }$ & & \\
\hline & Sehund & Euphorbia neriifolia L. & & & & & $\sqrt{ }$ & & \\
\hline & Shal-sarjarasa & Shorea robusta Gaertn. & $\sqrt{ }$ & & & & & & \\
\hline & Shallaki & Boswellia serrata Roxb. & & & & & $\sqrt{ }$ & & \\
\hline & Shan & Dioscorea polystachya Turcz. & $\sqrt{ }$ & & & & & & \\
\hline & Shigru & Moringa oleifera Lam. & & & $\sqrt{ }$ & & & & \\
\hline & Shinshapa & Dalbergia sissoo Roxb. ex DC. & & & $\sqrt{ }$ & & & & \\
\hline & Shyonak & Oroxylum indicum (L.) Kurz & & & & & $\begin{array}{l}\sqrt{ } \\
\text { (Co }\end{array}$ & 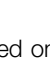 & \\
\hline
\end{tabular}


TABLE 1 | (Continued) List of plant and metal drugs as contraceptives in Ayurveda classics. Vertical column numbers indicate AaartavJanan - Emmenagogue (1), Aparapatan - placental expulsion (2), Garbhanuloman/Garbhapatkar-Abortifacient or Garbhastravakar-expel Fetus (3), Garbhanirodhak Contraceptives (4), Garbhashayasancochak-Ecbolic (5), Shandhyakar/Pumstvopadhatin- drugs that hamper male sexual or reproductive capability (6), and Shukrashodhan-Depurates (7).

\begin{tabular}{|c|c|c|c|c|c|c|c|c|c|}
\hline $\begin{array}{l}\text { Sr. } \\
\text { No. }\end{array}$ & $\begin{array}{l}\text { Sanskrit } \\
\text { name }\end{array}$ & $\begin{array}{l}\text { Botanical } \\
\text { name }\end{array}$ & (1) & (2) & (3) & (4) & (5) & (6) & (7) \\
\hline & Sitab & Ruta graveolens L. & & & & & $\sqrt{ }$ & $\sqrt{ }$ & $\sqrt{ }$ \\
\hline & Sitaphal & Annona squamosa L. & & & $\sqrt{ }$ & $\sqrt{ }$ & & & \\
\hline & Sunthi & Zingiber officinale Roscoe & & $\sqrt{ }$ & & & & & \\
\hline & Sweta Aparajita & Clitoria ternatea L. & & & $\sqrt{ }$ & & & & \\
\hline & Talisa patra & Abies spectabilis (D. Don) Mirb. & $\sqrt{ }$ & $\sqrt{ }$ & & $\sqrt{ }$ & & & \\
\hline & Tanduliyaka & Amaranthus spinosus L. & & & & $\sqrt{ }$ & & & \\
\hline & Tintidika & Tamarindus indica L. & & & & & & $\sqrt{ }$ & \\
\hline & Tilataila & Sesame oil & & & & $\sqrt{ }$ & & & \\
\hline & Tuvari & Cajanus cajan (L.) Huth & & & $\sqrt{ }$ & & & & \\
\hline & Ugra & Apium graveolens L. & & & $\sqrt{ }$ & & & & \\
\hline & Ulatakambal & Abroma augusta (L.) L.f. & $\sqrt{ }$ & & & & $\sqrt{ }$ & & \\
\hline & Unnab & Ziziphus jujuba Mill. & & & & & & $\sqrt{ }$ & \\
\hline & Upakunchika & Nigella sativa $L$. & & $\sqrt{ }$ & & $\sqrt{ }$ & $\sqrt{ }$ & & \\
\hline & Ushir & Chrysopogon zizanioides (L.) Roberty & & & & & & & $\sqrt{ }$ \\
\hline & Vacha & Acorus calamus L. & & & $\sqrt{ }$ & & & & \\
\hline & Vansha & Bambusa bambos (L.) Voss & $\sqrt{ }$ & & & & & $\sqrt{ }$ & \\
\hline & Vidanga & Embelia ribes Burm.f. & $\sqrt{ }$ & $\sqrt{ }$ & & $\sqrt{ }$ & & & \\
\hline & Visha & Aconitum chasmanthum Stapf ex Holmes & & & $\sqrt{ }$ & & & & \\
\hline \multicolumn{10}{|c|}{ Minerals/Metals } \\
\hline & Devalaya Churna & Scrapped lime powder from the wall of temple & & & & $\sqrt{ }$ & & & \\
\hline & Gairika & Red Ochre, $\mathrm{Fe}_{2} \mathrm{O}_{3}$ & & & & $\sqrt{ }$ & & & \\
\hline & Nausagar & $\mathrm{NH}_{4} \mathrm{Cl}$ & & & & & & $\sqrt{ }$ & \\
\hline & Saindhava lavana & Rock salt & & & & $\sqrt{ }$ & & & \\
\hline & Sindura & Lead oxide & & & $\sqrt{ }$ & $\sqrt{ }$ & & & \\
\hline & Tankana & Borax & & & & $\sqrt{ }$ & & & \\
\hline
\end{tabular}

along with Kanji (fermented drink), Tandulodaka (rice water), Sarkara (sugar candy), milk, and honey.

\section{POTENTIAL INGREDIENTS HAVING ANTIFERTILITY OR CONTRACEPTIVE PROPERTIES}

This literature survey revealed that there are about more than 94 indigenous medicinal plants having scientific evidence of acting as contraceptives. Some of the remarkable plant drugs with parts used, their chemical constituents, and pharmacological activities are described in Table 2. This compiled information will provide useful reference for new drug designing models, acting either as male or female contraceptives.

Pharmacologically, there are about 67 medicinal plants which possess antifertility activity in females and 56 medicinal plants in males. Several plants have shown to help contraception from the female and male perspectives.

In various experimental animal models, these herbal extracts have shown minimal side effects in comparison to the chemically synthesized contraceptives, which usually contain various combinations of hormones. These plant extracts have active phytoconstituents, which are responsible for the antifertility effects such as antiovulation, anti-implantation, and others.

\section{CLINICAL STUDIES}

Some of the plants that have demonstrated interesting antifertility activity in clinical trials are as follows.

\section{Embelia ribes Burm.f.}

Single drug was administered in a dose of $2^{\circ} \mathrm{g}$ for $5^{\circ}$ days followed by $1^{\circ} \mathrm{g}$ daily for another $10^{\circ}$ days. After observing the effect on 2051 cycles in 45 women over $4^{\circ}$ years, it was reported that the plant protected $95 \%$ of women from pregnancy (Tewari et al., 1976).

\section{Hibiscus rosa-sinensis $\mathrm{L}$.}

Red petals of the plant Rudrapushpaka collected between October and December. The extract was administered to 30 sexually active women at a dose of $750^{\circ} \mathrm{mg} /$ day from day 7 to day 22 of the reproductive cycle. It was observed that no one had become pregnant (Tewari, 1974).

\section{Neem oil}

A study was conducted on neem seed oil as local application for the reproductive female [ 246 women in the fertile age-group, 4 dropped out] as a method of family planning for a period of 12-36 cycles. In nine cases, there was conception due to drug failure and in four cases, there was conception due to drug omission. Neem seed oil may be used as an external barrier as 
TABLE 2 | Medicinal plants and their phyotoconstituents validated for various female/male contraceptive activities. Different contraceptive activities studied on medicinal plants could be categorized as follows. Female contraceptive activities: (2A) anti-implantation activity, (2B) abortification, (2C) antifertility, (2D) antiovulatory, and (2E) antiestrogenic activity. Male contraceptive activities: (2F) antispermatogenic, (2G) spermicidal, and (2H) antiandrogenic activity.

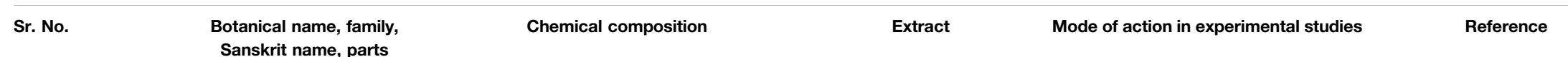

A Anti-implantation

activity

1.

3.

3.

4.

5.

Abies spectabilis (D. Don) Mirb.
Pinaceae
Talisa Patra, leaf
Abroma augusta (L.) L.f.
Malvaceae
Pishach karpas, roots
Adhatoda vasica Nees synonym
of Justicia adhatoda L.
Acanthaceae
Vasa, leaves
Ailanthus excelsa Roxb
Simaroubaceae
Maharukha, leaves
Allium cepa L.
Amaryllidaceae
Palandu, onion, bulb
Aloe barbadensis Mill.
Synonym of aloe vera (L.) Burm.f.
Asphodelaceae
Kumari, leaves
Areca catechu L.
Arecaceae
Poogaphala, Nuts
Cassia fistula L.
Fabaceae
Aragvadha, fruits, bark
Carica papaya L.
Caricaceae,
Papaya unripe fruit pulp, seeds,
latex
Centratherum anthelminticum
(L.) Gamble
Asteraceae
Vanya Jeeraka, seeds
Citrus $\times$ aurantium L
Rutaceae
Bijaura, seeds

Embelia ribes Burm.f.

Primulaceae

Vidang, berries
Flavonoids, bioflavonoids, glycosides, phytosterols

L-rhamnose, L-arabinose, D-xylose, D-

mannose, D-galactose, D-glucose, D-

galacturonic acid, and D-glucuronic acid

Alkaloids, tannins, saponins, and phenolics

flavonoids

Sitosterol, quassinoids, and ailantic acid

Kampferol, $\beta$-sitosterol, ferulic acid, and myritic Ethanolic acid

Water, polysaccharides, pectin, cellulose,

hemicellulose, and glucomannan

Alkaloids - pilocarpine, arecaidine, and arecoline

Alkaloid

\section{and aqueous}

Papain, caricacin, carpasemine, and oleanolic glycoside

Glycosides, carbohydrates, phenolic

compounds, tannins, flavonoids, proteins,

saponins, and sterols

Citroflavanoids, glucosides, and triterpenoids

Embelin, volatile oil, and fixed oil
Aqueous

Pet ether, alcohol, and

aqueous ethanol

Benzene, alcoholic

Alcoholic

Aqueous

Ethanolic and aqueous

(1)

Ethanol

Petroleum ether

Isolated embelin

Mode of action in experimental studies

Reference

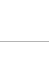


TABLE 2 | (Continued) Medicinal plants and their phyotoconstituents validated for various female/male contraceptive activities. Different contraceptive activities studied on medicinal plants could be categorized as follows. Female contraceptive activities: (2A) anti-implantation activity, (2B) abortification, (2C) antifertility, (2D) antiovulatory, and (2E) antiestrogenic activity. Male contraceptive activities: (2F) antispermatogenic, (2G) spermicidal, and $(2 \mathrm{H})$ antiandrogenic activity.

\begin{tabular}{|c|c|c|c|c|c|}
\hline Sr. No. & $\begin{array}{l}\text { Botanical name, family, } \\
\text { Sanskrit name, parts }\end{array}$ & Chemical composition & Extract & Mode of action in experimental studies & Reference \\
\hline 13. & $\begin{array}{l}\text { Gloriosa superba L. } \\
\text { Colchicaceae } \\
\text { Langli } \\
\text { Root }\end{array}$ & Colchicine (superbine) & $\begin{array}{l}\text { Hydroalcoholic extract at two } \\
\text { different doses }\end{array}$ & $\begin{array}{l}\text { Antifertility, anti-implantation activity in } \\
\text { postcoital study, abortifacient activity }\end{array}$ & Latha et al. (2013) \\
\hline 14. & $\begin{array}{l}\text { Grewia asiatica L. } \\
\text { Malvaceae, seeds }\end{array}$ & $\begin{array}{l}\text { Potassium, calcium, phosphorus, copper, zinc, } \\
\text { and magnesium }\end{array}$ & Aqueous & Anti-implantation and abortification activity & Kamboj and Dhawan (1982) \\
\hline 15. & $\begin{array}{l}\text { Hibiscus rosa-sinensis L. } \\
\text { Malvaceae } \\
\text { Japa } \\
\text { Flowers }\end{array}$ & Cyclopeptide alkaloid & Ethanol and benzene extract & $\begin{array}{l}\text { Anti-implantation, antiovulatory, increased } \\
\text { uterine weight, secretion of estrogenic by } \\
\text { atretic follicles, postcoital antifertility }\end{array}$ & $\begin{array}{l}\text { Neeru and Sharma (2008); } \\
\text { Vasudeva and Sharma (2008); } \\
\text { Hadimur et al. (2014), Pal et al. } \\
\text { (1985) }\end{array}$ \\
\hline 16. & $\begin{array}{l}\text { Mesua ferrea L. } \\
\text { Calophyllaceae } \\
\text { Nagakeshara, flowers }\end{array}$ & $\begin{array}{l}\text { Mesuol, mammegin, mesuaferronea, and } \\
\text { mammeuisin }\end{array}$ & Aqueous & Anti-implantation activity & $\begin{array}{l}\text { Seshadri and Pillai (1981); Munshi } \\
\text { et al. (1977) }\end{array}$ \\
\hline 17. & $\begin{array}{l}\text { Michelia champaca L. } \\
\text { Magnoliaceae } \\
\text { Champaka, Anthers }\end{array}$ & Essential oil & $\begin{array}{l}\text { Benzene and hydroalcholic } \\
\text { extract }\end{array}$ & Postcoital anti-implantation activity & $\begin{array}{l}\text { Sharma et al. (1994); Taprial et al. } \\
\text { (2013) }\end{array}$ \\
\hline 18. & $\begin{array}{l}\text { Momordica charantia L. } \\
\text { Cucurbitaceae } \\
\text { Karwellaka } \\
\text { roots, leaves }\end{array}$ & $\begin{array}{l}\text { Glycosides, saponins, alkaloids, fixed oils, } \\
\text { triterpenes, proteins, and steroids }\end{array}$ & Aqueous & $\begin{array}{l}\text { Uterine stimulant activity, Antifertility, } \\
\text { estrogenic activity }\end{array}$ & $\begin{array}{l}\text { Jamwal and Anand (1962); } \\
\text { Saksena (1971) }\end{array}$ \\
\hline 19. & $\begin{array}{l}\text { Plumbago zeylanica L. } \\
\text { Plumbaginaceae } \\
\text { Chitrak, root }\end{array}$ & Plumbagin & Plumbagin-free alcohol & Anti-implantation and abortifacient activity & Gupta et al. (2011) \\
\hline 20. & $\begin{array}{l}\text { Ricinus communis } \mathrm{L} \text {. } \\
\text { Euphorbiaceae } \\
\text { Erand, castor bean } \\
\text { Seed }\end{array}$ & Ricinine and isoquinoline & Aqueous & $\begin{array}{l}\text { Anti-implantation, increase in diameter of } \\
\text { the uterus, and decrease in uterine } \\
\text { hormones }\end{array}$ & Makonnen et al. (1999) \\
\hline 21. & $\begin{array}{l}\text { Rubia cordifolia L. } \\
\text { Rubiaceae } \\
\text { Manjishtha } \\
\text { Root }\end{array}$ & Munjistin, purpurin, and pseudopurpurin & Ethanolic extract & Anti-implantation & Maurya et al. (2004) \\
\hline 22. & $\begin{array}{l}\text { Sapindus trifoliatus L. } \\
\text { Sapindaceae } \\
\text { Arishtak } \\
\text { Fruits, pulp, and seeds }\end{array}$ & Essential oil & Butanol & $\begin{array}{l}\text { Antizygotic, blastocytotoxic, or anti- } \\
\text { implantation activity }\end{array}$ & $\begin{array}{l}\text { Pal et al. (2013); Bodhankar et al. } \\
\text { (1974) }\end{array}$ \\
\hline 23. & $\begin{array}{l}\text { Sesbania sesban (L.) Merr. } \\
\text { Fabaceae } \\
\text { Sesban } \\
\text { Leaves }\end{array}$ & $\begin{array}{l}\text { Alkaloids, flavonoids, glycosides, tannin, } \\
\text { anthraquinone, steroid, pholobatannins, and } \\
\text { terpenoids }\end{array}$ & Extract and powder & $\begin{array}{l}\text { Inhibit the ovarian function, change the } \\
\text { uterine structure, and prevent the } \\
\text { implantation }\end{array}$ & $\begin{array}{l}\text { Singh (1990a); Samajdar and } \\
\text { Ghosh (2017) }\end{array}$ \\
\hline \multicolumn{6}{|l|}{$\begin{array}{l}\text { B Abortification } \\
\text { activity }\end{array}$} \\
\hline 1. & $\begin{array}{l}\text { Abroma augusta (L.) L.f. } \\
\text { Malvaceae } \\
\text { Pishach karpas, roots }\end{array}$ & $\begin{array}{l}\text { L-rhamnose, L-arabinose, D-xylose, } \\
\text { D-mannose, D-galactose, D-glucose, } \\
\text { D-galacturonic acid, and D-glucuronic acid }\end{array}$ & Alcoholic & Abortification activity & $\begin{array}{l}\text { Pokharkar et al. (2010); Kalita et al. } \\
\text { (2011) }\end{array}$ \\
\hline
\end{tabular}


TABLE 2 | (Continued) Medicinal plants and their phyotoconstituents validated for various female/male contraceptive activities. Different contraceptive activities studied on medicinal plants could be categorized as follows. Female contraceptive activities: (2A) anti-implantation activity, (2B) abortification, (2C) antifertility, (2D) antiovulatory, and (2E) antiestrogenic activity. Male contraceptive activities: (2F) antispermatogenic, (2G) spermicidal, and $(2 \mathrm{H})$ antiandrogenic activity.

Chemical composition

Extract

Mode of action in experimental studies

Reference

Abrus precatorius L.

Abrin, abrasine, precasine, and precol

Aqueous

Fatty acids, oleonic acid, bisdesmosidic,

Benzene, ethanolic, and

Achyranthes aspera L.

chloroform

Amaranthaceae

ApamargaWhole plant,

Stem bark, Root

of Justicia adhatoda achyranthine

Acanthaceae, Vasa, Leaves

Aegle marmelos (L) Corrêa

Aegle marmelos (L.) Corrêa.

Bilva, whole plant, leaves

6. Annona squamosa L.

Annonaceae

Custard apple

Seeds, leaves, and bark

7. Areca catechu L.

Areca catechu

Poogaphala, nuts

8. Barleria prionitis L.

Acanthaceae

Saireyak, Roots

9. Carica papaya L

Caricaceae

Papaya unripe fruit pulp,

seeds, and latex

Citrus $\times$ aurantium $L$

Rutaceae

Bijaura, Seeds

$11 . \quad$ Daucus carota L.

Apiaceae

Grinjanak, seed

Gloriosa superba L.

Colchicaceae

Langli

Root

$13 . \quad$ Grewia asiatica L.

Malvaceae, seeds

Brassicaceae

Chandrasur

Mature explants

Alkaloids, tannins, saponins, phenolics, and Aqueous flavonoids

Marmelosin, luvangetin, psoralen, tannins, and Aqueous extract marmin

Atropine alkaloids, and anonaine

Ethyl acetate extract

Alkaloids-pilocarpine, arecaidine, and arecoline Petroleum ether, alcoholic, and aqueous

Acbarlerin, barlerin, B-sitosterol, flavanol Methanol extract

glycoside, and iridoids

Papain, caricacin, carpasemine, and oleanolic Pet ether, alcohol, and glycoside,

aqueous ethanol

Citroflavanoids, glucosides, and triterpenoids Petroleum ether

Essential oil

roleum, ether, benzene,

alcohol, and water

Carbohydrates, flavonoids, steroids, alkaloids, tannins, and glycosides

ther, chloroform, and ethy alcohol extracts

Potassium, calcium, phosphorus, copper, zinc, Aqueous

and magnesium

Lepidine

Methanolic bortifacient activity or antifertility agent with

a risk of DNA damage

ortifacient activity in rabbits

Sarwat et al. (2009); Kaur et al. (2011); Shrivastava et al. (2007); Azmeera et al. (2012); Priya et al. (2012)

Raj et al. (2011); Vasudeva and

Sharma (2006)

Abortification activity

Pokharkar et al. (2010); Kaur et al. (2011); Raj et al. (2011)

Abortifacient activity in albino rats

Gangadhar and Lalithakumar (1995); Sathiyaraj et al. (2010)

Abortifacient induces early abortion

Jain and Dixit (1992)

Abortifacient activity in albino rats and antifertility activity

Abortifacient

Garg and Garg (1970); Garg and Garg (1971); Shrestha et al. (2010)

Gupta et al. (2000)

Abortifacient in albino rats and antifertility

Garg and Garg (1970), Garg and Garg (1971); Das (1980); Sinha and Nathawat (1989);

Changamma and Lakshman

(2013)

Abortifacient, increased ovarian weight, decreased Graafian follicles, and irregular estrous cycle

Abortifacient activity

Patil and Patil (2013)

Garg (1975); Jansen and Wolhlmuth (2014); Shah and Varute (1980)

Abortifacient activity and significant reduction in number of implants and number of pups born

Abortification activity

Malpani and Mahurkar (2018)

Abortifacient and antiovulatory
Kamboj and Dhawan (1982)

Pande et al. (2002) 
TABLE 2 | (Continued) Medicinal plants and their phyotoconstituents validated for various female/male contraceptive activities. Different contraceptive activities studied on medicinal plants could be categorized as follows. Female contraceptive activities: (2A) anti-implantation activity, (2B) abortification, (2C) antifertility, (2D) antiovulatory, and (2E) antiestrogenic activity. Male contraceptive activities: (2F) antispermatogenic, (2G) spermicidal, and $(2 \mathrm{H})$ antiandrogenic activity. Sanskrit name, parts

Chemical composition

Extract

Mode of action in experimental studies

Reference

Ricinus communis L.

Ricinine and isoquinoline

Aqueous extract

Abortifacient

Makonnen et al. (1999),

Erand, Castor bean

Seed

Woodfordia fruticosa (L.) Kurz

Lythraceae

C Antifertility activity

Dhataki, flowers

Abrus precatorius $\mathrm{L}$.

Papilionaceae

Gunja, Seeds

Acacia leucophloea (Roxb.) Willd.

Leguminosae-Fabaceae

Shwet babul, roots

3. Annona squamosa L.

Annonaceae

Custard apple

Seeds, leaves, and bark

4. Areca catechu L.

Arecaceae

Poogaphala, Nuts

Azadirachta indica A. Juss

Meliaceae

Nimba

Leaves, flower, and seed

Carica papaya L.

Caricaceae

Papaya unripe fruit pulp

seeds, and latex

Cissampelos pareira L.

Menispermaceae, Patha

8.

Leaves and stem

Cuminum cyminum L

Apiaceae

Jeerak, seeds

9. Crateva nurvala Buch-Ham.

Capparaceae

Varuna

10.

Dried stem bark

Curcuma longa L.

Zingiberaceae

Tannins, flavonoids, anthraquinone glycosides, Aqueous and ethanol and polyphenols

Abrin, abrasine, precasine, and precol

Aqueous

Antifertility agent with a risk of DNA damage

Sarwat et al. (2009); Kaur et al. 2011); Shrivastava et al. (2007); Azmeera et al. (2012); Priya et a (2012)

hexacosanol, beta-amyrin, beta-sitosterol, Alcoholic and tannin

Atropine alkaloids and anonaine

Ethyl acetate extract

Antifertility activity

Dheeraj (2011)

Alkaloids - pilocarpine, arecaidine, and arecoline Nut oil Ethanolic extract

Antifertility activity in female albino rats,

antiovulatory, and ovarian weight

decreased due to imbalance in

gonadotrophins

Azadirachtin, nimbolinin, nimbin, nimbidin, nimbidol, sodium nimbinate, and gedunin

Papain, caricacin, carpasemine, and oleanolic glycoside

Pet ether, alcohol, aqueous,

Antifertility

and ethano

Leaf extract

Berberine

Extract

ered the estrous cycle pattern in female mice, Antifertility

Cuminal and cuminic alcoho

Antifertility effect in female albino rat

Alkaloids, triterpene, tannins, saponins,

flavonoids, sterols, glucosylinate, lupeol, and

Ethanol, aqueous

Antifertility effects estrogenic activity

2010)

Vyas and Purohit (2018)

diosgenin

Curcumin and flavanoids

Ethanol, aqueous

Propylene glycol solution, antifertility, antiovulatory-suppression of GnRH

Ghosh et al. (2011); Bhagat and Purohit (1986)

(Continued on following page)

Garg and Garg (1970); Garg and Garg (1971); Das (1980); Sinha and Nathawat (1989):

Changamma and Lakshman (2013)

al. (2011)

Priya et al. (2012); Sharma J et al. (2001)

Bhaskar et al. (2009) 
TABLE 2 | (Continued) Medicinal plants and their phyotoconstituents validated for various female/male contraceptive activities. Different contraceptive activities studied on medicinal plants could be categorized as follows. Female contraceptive activities: (2A) anti-implantation activity, (2B) abortification, (2C) antifertility, (2D) antiovulatory, and (2E) antiestrogenic activity. Male contraceptive activities: (2F) antispermatogenic, (2G) spermicidal, and $(2 \mathrm{H})$ antiandrogenic activity.

\begin{tabular}{|c|c|c|c|c|c|}
\hline Sr. No. & $\begin{array}{l}\text { Botanical name, family, } \\
\text { Sanskrit name, parts }\end{array}$ & Chemical composition & Extract & Mode of action in experimental studies & Reference \\
\hline 11. & $\begin{array}{l}\text { Daucus carota L. } \\
\text { Apiaceae } \\
\text { Grinjanak, Seed }\end{array}$ & Essential oil & $\begin{array}{l}\text { Petroleum, ether, benzene, } \\
\text { alcohol, and water }\end{array}$ & Antifertility activity & $\begin{array}{l}\text { Garg (1975); Jansen and } \\
\text { Wolhlmuth (2014); Shah and } \\
\text { Varute (1980) }\end{array}$ \\
\hline 12. & $\begin{array}{l}\text { Desmodium gangeticum (L.) DC. } \\
\text { Fabaceae } \\
\text { Shaliparni, Root }\end{array}$ & $\begin{array}{l}\text { Lavonoid glycosides, pterocarpanoids, lipids, } \\
\text { glycolipids, and alkaloids }\end{array}$ & Gangeticum & Antifertility effect & Pillai et al. (1982) \\
\hline 13. & $\begin{array}{l}\text { Embelia ribes Burm.f. } \\
\text { Primulaceae } \\
\text { Vidang, Berries }\end{array}$ & $\begin{array}{l}\text { Embelin, volatile oil, fixed oil, resin, tannin, } \\
\text { christembine (alkaloid), and phenolic acids }\end{array}$ & Isolated embelin & $\begin{array}{l}\text { Anti-implantation and postcoital antifertility } \\
\text { activity }\end{array}$ & Prakash (1981b) \\
\hline 14. & $\begin{array}{l}\text { Ferula jaeschkeana Vatke } \\
\text { Apiaceae } \\
\text { Heengupatri, } \\
\text { Dried leaves }\end{array}$ & $\begin{array}{l}\text { Flavonoids, alkaloids, terpenoids, cardiac } \\
\text { glycosides, saponins, and phenolics }\end{array}$ & Hexane & $\begin{array}{l}\text { Duration-dependent luteolytic changes in } \\
\text { the corpora lutea }\end{array}$ & Pathak et al. (1995) \\
\hline 15. & $\begin{array}{l}\text { Gloriosa superba L. } \\
\text { Colchicaceae } \\
\text { Langli, Root }\end{array}$ & Colchicine (superbine) & $\begin{array}{l}\text { Hydroalcoholic extract at two } \\
\text { different doses } 30 \text { and } \\
60^{\circ} \mathrm{mg} / \mathrm{kg}\end{array}$ & $\begin{array}{l}\text { Antifertility, anti-implantation activity in } \\
\text { postcoital study }\end{array}$ & Latha et al. (2013) \\
\hline 16. & $\begin{array}{l}\text { Hibiscus rosa-sinensis L. } \\
\text { Malvaceae } \\
\text { Japa } \\
\text { Flowers }\end{array}$ & Cyclopeptide alkaloid & Ethanol and benzene extract & $\begin{array}{l}\text { Anti-implantation, antiovulatory, secretion of } \\
\text { estrogenic by atretic follicles, and postcoital } \\
\text { antifertility }\end{array}$ & Neeru and Sharma (2008) \\
\hline 17. & $\begin{array}{l}\text { Lawsonia inermis L. } \\
\text { Lythraceae } \\
\text { Madayantika } \\
\text { Leaves }\end{array}$ & $\begin{array}{l}\text { Lawsone, esculetin, fraxetin, isoplumbagin, } \\
\text { scopoletin, betulin, betulinic acid, hennadiol, } \\
\text { lupeol, lacoumarin, quinone, and napthaquinone }\end{array}$ & Powder & $\begin{array}{l}\text { Preventing pregnancy in } 60 \% \text { of the animals } \\
\text { tested }\end{array}$ & Munshi et al. (1977) \\
\hline 18. & $\begin{array}{l}\text { Lepidium sativum } L \text {. } \\
\text { Brassicaceae } \\
\text { Chandrasur } \\
\text { Mature explants }\end{array}$ & Lepidine & Methanolic & Abortifacient and antiovulatory & Pande et al. (2002) \\
\hline 19. & $\begin{array}{l}\text { Melia azedarach L. Meliaceae, } \\
\text { MalaiVembu } \\
\text { seed and leaves }\end{array}$ & Triterpenoids & Seed extract & $\begin{array}{l}\text { Antifertility effect, increased } \\
\text { preimplantation, postimplantation, and total } \\
\text { prenatal mortalities }\end{array}$ & Mandal and Dhariwal (2007) \\
\hline 20. & $\begin{array}{l}\text { Momordica charantia L. } \\
\text { Cucurbitaceae } \\
\text { Karwellaka } \\
\text { Roots and leaves }\end{array}$ & $\begin{array}{l}\text { Glycosides, saponins, alkaloids, fixed oils, } \\
\text { triterpenes, proteins, and steroids }\end{array}$ & Aqueous & $\begin{array}{l}\text { Uterine stimulant activity, antifertility, and } \\
\text { estrogenic activity }\end{array}$ & $\begin{array}{l}\text { Jamwal and Anand (1962); } \\
\text { Saksena (1971) }\end{array}$ \\
\hline 21. & $\begin{array}{l}\text { Nigella sativa } L \text {. } \\
\text { Ranunculaceae } \\
\text { Krishna jeerak, Seeds }\end{array}$ & Fixed oil, volatile oil, and alkaloids & Hexane & $\begin{array}{l}\text { Antifertility activity in rats, postcoital } \\
\text { contraceptive }\end{array}$ & Keshri et al. (1995) \\
\hline 22. & $\begin{array}{l}\text { Piper betle L } \\
\text { Piperaceae } \\
\text { Betel leaf, Pan } \\
\text { Petiol }\end{array}$ & $\begin{array}{l}\text { Eugenol, eugenol acetate, piper betol, piperol, } \\
\text { and methyl eugenol phytol }\end{array}$ & Alcoholic & $\begin{array}{l}\text { Antifertility, antiestrogenic effects in female } \\
\text { rats }\end{array}$ & Sharma et al. (2007) \\
\hline 23. & $\begin{array}{l}\text { Piper longum L. } \\
\text { Piperaceae } \\
\text { Pippali } \\
\text { Root and ruits }\end{array}$ & Piperine & $\begin{array}{l}\text { Powder, hexane fraction, and } \\
\text { benzene }\end{array}$ & $\begin{array}{l}\text { Antifertility activity-prolonged the length of } \\
\text { the extort cycle, drastic reduction in the } \\
\text { number of implantation sites, marked } \\
\text { suppression in the ovarian cytokines and } \\
\text { nitric acid level }\end{array}$ & $\begin{array}{l}\text { Laxmi et al. (2006); Kholkute et al. } \\
\text { (1979) }\end{array}$ \\
\hline
\end{tabular}


TABLE 2 | (Continued) Medicinal plants and their phyotoconstituents validated for various female/male contraceptive activities. Different contraceptive activities studied on medicinal plants could be categorized as follows. Female contraceptive activities: (2A) anti-implantation activity, (2B) abortification, (2C) antifertility, (2D) antiovulatory, and (2E) antiestrogenic activity. Male contraceptive activities: (2F) antispermatogenic, (2G) spermicidal, and $(2 \mathrm{H})$ antiandrogenic activity.

otanical name, family

Chemical composition

Extract

Mode of action in experimental studies

Reference

Trichosanthes cucumerina L.

Cucurbitacin $B$, cucurbitacin $E$, isocucurbitacin Aqueous

$\mathrm{B}, \mathrm{E}$, sterols $2 \beta$-sitosterol stigmasterol

Affected the normal estrous cycle,

Devendra et al. (2009)

Snake gourd, Fruit

significantly reduced the number of healthy

follicles, corpora lutea, and increased the

number of regressing follicles. Reduced

25.

Zingiber officinale

Monocyclic, phenols, sesquiterpenees sential oil, Aqueous, ethanol extracts

oleoresins, and proteolytic enzymes

Antifertility activity

Pathak et al. (2005)

Zingiberaceae

Sunthi

D Antiovulatory

activity

Poogaphala, Nuts

Azadirachta indica A. Juss. Azadirachtin, nimbolinin, nimbin, nimbidin,

Meliaceae

Achyranthes aspera L.

Amaranthaceae

ApamargaWhole plant,

Stem bark, Root

Leaves, flower, and seed

4. Butea monosperma (Lam)

Kuntze

Fabaceae

Palash, bark, and flowers

Calotropis procera (Aiton) W.T. Steroidal alkaloid

Fatty acids, oleonic acid, bisdesmosidic

Benzene, ethanolic

triterpenoid alkaloids, D-glucuronic, betaine, and chloroform

achyranthine

Alkaloids - pilocarpine, arecaidine, and arecoline Ethanolic extract

nimbidol, sodium nimbinate, and gedunin

Alcoholic extract flower in

Sprague-Dawley rats

Kino-tannic acid, gallic acid, and pyrocatechin Aqueous extract

Calotropin, aqueous ethanol

Aiton

Apocynaceae

Catunaregam spinosa (Thunb.) Saponins, valeric acid resin, wax, and coloring Ethanolic extract, isolated oleic

Tiveng.

matter

Madanphal,

Fruits, seeds, and pulp

Citrus $\times$ aurantium

Rutaceae

Bijaura, Seeds

8.

Curcuma longa L.

Zingiberaceae

Haldi, rhizome

9. Hibiscus rosa-sinensis $\mathrm{L}$.

Malvaceae

Japa, Flowers

Citroflavanoids, glucosides, and triterpenoids Petroleum ether

Curcumin and flavanoids

Ethanol, aqueous

Cyclopeptide alkaloid

Ethanol, benzene extract
Antiovulatory prolonged di-estrous stage

with temporary inhibition of ovulation

Antiadulaton, anti-implantation, hormon disturbance in uterus, and expulsion of ovary

Antiovulatory, ovarian weight decreased due to imbalance in gonadotrophins

Disrupted the estrous cycle and caused a partial block in ovulation

Inhibit ovulation

Antiovulatory effect in rabbits,

antiimplantation activity in albino rats

Anti-implantation, antiovulatory,

abortifacient, increased ovarian weight,

decreased Graafian follicles, irregular

estrous cycle

Propylene glycol solution antifertility,

antiovulatory, decreased ovarian weight,

suppression of $\mathrm{GnRH}$

Anti-implantation, antiovulatory, increased

uterine weight, secretion of estrogenic by

atretic follicles, postcoital antifertility
Shibeshi ot al (2006): Vasudeva and Sharma (2006)

Shrestha et al. (2010)

Gbotolorun et al. (2003); Vyas an Purohit (2018)

Shrivastava et al. (2007), Sinha and Nathawat (1989)

Gupta et al. (1990); Abdelgader and Elsheikh (2018); Sharma and Jacob (2001a): Pokharkar et al. (2010)

Malhi and Trivedi (1972); Pillai et al. (1977)

Patil and Patil (2013)

Ghosh et al. (2011)

Neeru and Sharma (2008)

(Continued on following page) 
TABLE 2 | (Continued) Medicinal plants and their phyotoconstituents validated for various female/male contraceptive activities. Different contraceptive activities studied on medicinal plants could be categorized as follows. Female contraceptive activities: (2A) anti-implantation activity, (2B) abortification, (2C) antifertility, (2D) antiovulatory, and (2E) antiestrogenic activity. Male contraceptive activities: (2F) antispermatogenic, (2G) spermicidal, and $(2 \mathrm{H})$ antiandrogenic activity.

\begin{tabular}{|c|c|c|c|c|c|}
\hline Sr. No. & $\begin{array}{l}\text { Botanical name, family, } \\
\text { Sanskrit name, parts }\end{array}$ & Chemical composition & Extract & Mode of action in experimental studies & Reference \\
\hline 10. & $\begin{array}{l}\text { Musa paradisiaca L. } \\
\text { Musaceae, Banana, stem }\end{array}$ & Alkaloids and flavonoids & Ethanolic & $\begin{array}{l}\text { Antiovulatory suppressed ovulation due to } \\
\text { inhibition in secretion of GnRH }\end{array}$ & Soni et al. (2013) \\
\hline 11. & $\begin{array}{l}\text { Papaver somniferum L. } \\
\text { Papaveraceae } \\
\text { Ahiphen, Latex }\end{array}$ & Noscapine alkaloid & Alcoholic extract & $\begin{array}{l}\text { Antiovulatory decreased production of } \\
\text { gonadotrophin }\end{array}$ & Kumar and Sachin (2013) \\
\hline 12. & $\begin{array}{l}\text { Plumbago rosea L. } \\
\text { Plumbaginaceae } \\
\text { Raktachitrak, Leaves }\end{array}$ & $\begin{array}{l}\text { Plumbagin, sitosterol glycoside, tannins, and } \\
\text { fatty alcohol }\end{array}$ & Acetone, ethanolic & $\begin{array}{l}\text { Antiovulatory inhibition of ovulation with } \\
\text { irregular estrous cycle }\end{array}$ & Sheeja et al. (2011) \\
\hline 13. & $\begin{array}{l}\text { Semecarpus anacardium L.f. } \\
\text { Anacardiaceae } \\
\text { Bhallatak } \\
\text { Fruits }\end{array}$ & Alkaloids & Aqueous and ethanolic & Reversible antiovulatory activity & Sushma et al. (2016) \\
\hline 14. & $\begin{array}{l}\text { Taxus baccata L. } \\
\text { Taxaceae } \\
\text { Talishpatra Common Yew } \\
\text { Leaves }\end{array}$ & Pseudo alkaloids & Leaf extract & $\begin{array}{l}\text { Antiovulatory, inhibited secretion of ovarian } \\
\text { hormones }\end{array}$ & $\begin{array}{l}\text { Priya et al. (2012); Kaur et al. } \\
\text { (2011) }\end{array}$ \\
\hline 15. & $\begin{array}{l}\text { Vitex negundo L. } \\
\text { Lamiaceae } \\
\text { Nirgundi, roots, and seeds }\end{array}$ & $\begin{array}{l}\text { Casticin, isoorientin, chrysophenol D, luteolin, } \\
\text { p-hydroxybenzoic acid, and D-fructose }\end{array}$ & Aqueous & Antiovulatory activity & Lal et al. (1992) \\
\hline \multicolumn{6}{|l|}{$\begin{array}{l}\text { E Antiestrogenic } \\
\text { activity }\end{array}$} \\
\hline 1. & $\begin{array}{l}\text { Allium sativum } \mathrm{L} \text {. } \\
\text { Amaryllidaceae } \\
\text { Rason, Bulb }\end{array}$ & Sulfur-containing compounds & Alcohol & $\begin{array}{l}\text { Ecobolic in mice and rats, estrogenic activity } \\
\text { in female albino rats }\end{array}$ & $\begin{array}{l}\text { Tewari et al. (1971); Ola-Mudathir } \\
\text { et al. (2008) }\end{array}$ \\
\hline 2. & $\begin{array}{l}\text { Cyperus rotundus L. } \\
\text { Cyperaceae } \\
\text { Musta, Rhizome }\end{array}$ & $\begin{array}{l}\text { Cyperene, humulen, selinene, zierone, } \\
\text { campholenicopaene, and limonene }\end{array}$ & Aqueous & Antiestrogenic property & Gediya et al. (2011) \\
\hline 3. & $\begin{array}{l}\text { Glycyrrhiza glabra L. } \\
\text { Fabaceae } \\
\text { Yashtimadhu, Roots }\end{array}$ & Triterpene glycyrrhizin acid and glycoside & Water & Estrogenic activity & Ahmad et al. (2011) \\
\hline 4. & $\begin{array}{l}\text { Guilandina bonduc L. sy. } \\
\text { Caesalpinia bonduc (L.) Roxb. } \\
\text { Leguminosae } \\
\text { Karanja, seeds }\end{array}$ & $\begin{array}{l}\text { Phytosterinin, } \beta \text {-sitosterol, flavonoids, } \\
\text { bonducellin, aspartic acid, arginine, and citrulline } \\
\beta \text {-carotene }\end{array}$ & Aqueous & Antiestrogenic activity & Salunke et al. (2011) \\
\hline 5. & $\begin{array}{l}\text { Nelumbo nucifera Gaertn. } \\
\text { Nelumbonaceae } \\
\text { Kamala, Lotus } \\
\text { Seeds }\end{array}$ & Hydrocarbons & Ethanolic extract & $\begin{array}{l}\text { Antiestrogenic, decreased ovarian weight, } \\
\text { estrogens inhibition }\end{array}$ & Mutreja et al. (2008) \\
\hline 6. & $\begin{array}{l}\text { Sesamum indicum } L \text {. } \\
\text { Pedaliaceae } \\
\text { Tila, seeds }\end{array}$ & Oil, protein, and carbohydrate & Extract & Estrogenic effect in female albino rats & Priya et al. (2012) \\
\hline 7. & $\begin{array}{l}\text { Vitex negundo L. } \\
\text { Lamiaceae } \\
\text { Nirgundi, roots and seeds }\end{array}$ & $\begin{array}{l}\text { Casticin, isoorientin, chrysophenol D, luteolin, } \\
\text { p-hydroxybenzoic acid, and D-fructose }\end{array}$ & Aqueous & Antiovulatory activity & Lal et al. (1992) \\
\hline
\end{tabular}


TABLE 2 | (Continued) Medicinal plants and their phyotoconstituents validated for various female/male contraceptive activities. Different contraceptive activities studied on medicinal plants could be categorized as follows. Female contraceptive activities: (2A) anti-implantation activity, (2B) abortification, (2C) antifertility, (2D) antiovulatory, and (2E) antiestrogenic activity. Male contraceptive activities: (2F) antispermatogenic, (2G) spermicidal, and $(2 \mathrm{H})$ antiandrogenic activity.

$\begin{array}{cccc}\text { Sr. No. } & \begin{array}{c}\text { Botanical name, family, } \\ \text { Sanskrit name, parts }\end{array} & \text { Chemical composition } & \text { Extract }\end{array}$

F Antispermatogenic

activity

1.

Abru sprecatorius L.
Papilionaceae

Gunja, seeds

2.

Aegle marmelos (L.) Corrêa

Rutaceae

Bilva, whole plant and leaves

3.

Albizia lebbeck (L.) Benth.

Fabaceae

Shirish, Pods

4. Andrographis paniculat

(Burm.f.) Nees

Acanthaceae

5. $\quad$ Ananas comosus (L.) Merr.

Bromeliaceae
Custard apple, seeds

Annona squamosa L.

Annonaceae

Custard apple

Seeds, leaves, and bark

Areca catechu L.

Arecaceae

Poogaphala, Nuts

8. Aristolochia indica L.

Aristolochiaceae

Ishwari, roots

9.

Azadirachta indica A. Juss.

Meliaceae

Nimba

Leaves, flower, and seed

$10 . \quad$ Bacopa monnieri (L.) Wettst.

Plantaginaceae

Brahmi, whole plant

11. Balanites roxburghii

Planch.

Zygophyllaceae

Ingudi, Fruit pulp
Abrin, abrasine, precasine, and precol

Marmelosin, luvangetin, psoralen, tannins, and Aqueous extract marmin

Melacacidin, D-catechin, $\beta$-sitosterol, albiziahexoside, betulnic acid, and echinocystic acid glycosides

Andrographolide, Andrographidoids A, B, C, D, Water extract

E, diterpenoid, and lactone

Atropine alkaloids and anonaine

Atropine alkaloids and anonaine

Aristolochic acid, ceryl alcohol, $\beta$-sitosterol

stigmast-4-en-3-one, friedelin, and

cycloeucalenol

Azadirachtin, nimbolinin, nimbin, nimbidin,

nimbidol, sodium nimbinate, and gedunin

Bacosides and saponins

Saponin, furanocoumarin, and flavonoid

Berberine and berbamine
Aqueous

Water

Ethyl acetate extract

Antispermatogenic activity

No abnormality in Leydig cell and

interstitium tissue

Antispermatogenic

Reduced sperm motility, density,

antispermatogenic effect, reduced activity

enticular enzyme, post-testicular

antifertility effect

Inhibit spermatogenesis and sperm motility uction, affecting the sexual

ond epididymal sperm

Spermatogenic arrest in male albino rats

Antispermatogenic

Antispermatogenic activity

Decrease in the weight of seminal vesicles, ventral prostate, reduction in epithelial

height, nuclear diameter, and the secretory

materials in the lumen

Reversible suppression of spermatogenesis and fertility, without producing apparent toxic effects

Methanol, palmitine hydroxide Antispermatogenic activity

Antispermatogenic action
Bajaj et al. (1981); Dixit et al. (1987); Kulshreshtha and Mathur

(1990); Sinha (1990)

Sur et al. (1999); Sur et al. (2002

Gupta et al. (2004); (Gupta et al. 2005a)

Akbarsha et al. (1990); Akbarsha and Murugaian (2000)

Satyawati (1983)

Jain and Dixit (1992)

Ave Olivia et al. (2020)

Gupta et al. (1996)

Gediya et al. (2011)

Singh et al. (2013)

Dixit et al. (1981), Agarwal and Dixit (1982)

Gupta and Dixit (1989)

(Continued on following page) 
TABLE 2 | (Continued) Medicinal plants and their phyotoconstituents validated for various female/male contraceptive activities. Different contraceptive activities studied on medicinal plants could be categorized as follows. Female contraceptive activities: (2A) anti-implantation activity, (2B) abortification, (2C) antifertility, (2D) antiovulatory, and (2E) antiestrogenic activity. Male contraceptive activities: (2F) antispermatogenic, (2G) spermicidal, and $(2 \mathrm{H})$ antiandrogenic activity.

\begin{tabular}{|c|c|c|c|c|c|}
\hline Sr. No. & $\begin{array}{l}\text { Botanical name, family, } \\
\text { Sanskrit name, parts }\end{array}$ & Chemical composition & Extract & Mode of action in experimental studies & Reference \\
\hline 13. & $\begin{array}{l}\text { Butea monosperma (Lam.) } \\
\text { Kuntze } \\
\text { Fabaceae } \\
\text { Palash, bark, and flowers }\end{array}$ & Kino-tannic acid, gallic acid, and pyrocatechin & Aqueous extract & Antispermatogenic effect & Wati and Verute (1988) \\
\hline 14. & $\begin{array}{l}\text { Calotropis procera (Aiton) W.T. } \\
\text { Aiton } \\
\text { Apocynaceae } \\
\text { Arka, root }\end{array}$ & Steroidal alkaloid & Calotropin, aqueous ethanol & $\begin{array}{l}\text { Antispermatogenic, antiandrogenic } \\
\text { activities, and/or endocrine disrupting } \\
\text { effects, functional alteration in genital organ }\end{array}$ & $\begin{array}{l}\text { Gupta et al. (1990); Abdelgader } \\
\text { and Elsheikh (2018); Sharma and } \\
\text { Jacob (2001b) Pokharkar et al. } \\
\text { (2010) }\end{array}$ \\
\hline 15. & $\begin{array}{l}\text { Carica papaya } L \text {. } \\
\text { Caricaceae } \\
\text { Papaya, unripe fruit pulp, } \\
\text { seeds, latex }\end{array}$ & $\begin{array}{l}\text { Papain, caricacin, carpasemine, oleanolic } \\
\text { glycoside, }\end{array}$ & $\begin{array}{l}\text { Pet ether, Alcohol, aqueous } \\
\text { Ethanol }\end{array}$ & $\begin{array}{l}\text { Antispermatogenic activity reduced } \\
\text { spermatogenesis, inhibition in steroidal } \\
\text { hormones }\end{array}$ & $\begin{array}{l}\text { Changamma and Lakshman } \\
\text { (2013) }\end{array}$ \\
\hline 16. & $\begin{array}{l}\text { Celastrus paniculatus } \\
\text { Will. } \\
\text { Celastraceae } \\
\text { Jyotishmati, seeds }\end{array}$ & $\begin{array}{l}\text { Alkaloids, tannins, saponins, steroid, terpenoid, } \\
\text { flavonoids, phlobatannin, cardiac, and glycoside }\end{array}$ & Seed & Antispermatogenic activity & Bidwai et al. (1990) \\
\hline 17. & $\begin{array}{l}\text { Cichorium intybus L. } \\
\text { Asteraceae, Chicory } \\
\text { Whole plant }\end{array}$ & $\begin{array}{l}\text { Inulin, sesquiterpene lactones, vitamins, } \\
\text { minerals, fat, and mannitol, }\end{array}$ & Aqueous & Antispermatogenic activity & Roy and Venkatakrishna (1983) \\
\hline 18. & $\begin{array}{l}\text { Cinnamomum camphora (L.) } \\
\text { J.Presl } \\
\text { Lauraceae } \\
\text { Karpur } \\
\text { Camphor, leaves and resin }\end{array}$ & Essential oil-camphor, linalool, and cineole & Leaf & Inhibition of spermatogenesis & Singh (1990b) \\
\hline 19. & $\begin{array}{l}\text { Cuminum cyminum } L \\
\text { Apiaceae } \\
\text { Jeerak, seeds }\end{array}$ & Cuminal and cuminic alcohol & Extract & Antispermatogenic effect & $\begin{array}{l}\text { Priya et al. (2012); Sharma J et al. } \\
\text { (2001) }\end{array}$ \\
\hline 20. & $\begin{array}{l}\text { Embelia ribes Burm.f. } \\
\text { Primulaceae } \\
\text { Vidang, berries }\end{array}$ & Embelin, volatile oil, and fixed oil & Isolated embelin & Inhibition of spermatozoa motility & $\begin{array}{l}\text { Prakash (1981); Nand (1981); Dixit } \\
\text { et al. (1983); Gupta et al. (1989) }\end{array}$ \\
\hline 21. & $\begin{array}{l}\text { Euphorbia neriifolia L. } \\
\text { Milk brush } \\
\text { Euphorbiaceae } \\
\text { Latex, Whole plant }\end{array}$ & $\begin{array}{l}\beta \text {-amyrin acetate, lupenone, 3-acetoxy-20- } \\
\text { lupanol, cycloart-25-en-3 } 3,24 \zeta \text {-diol, and } \\
\text { cycloart }\end{array}$ & Ethanol & Antispermatogenic effect & Mali (1999) \\
\hline 22. & $\begin{array}{l}\text { Hibiscus rosa-sinensis L. } \\
\text { Malvaceae } \\
\text { Japa } \\
\text { Flowers }\end{array}$ & Cyclopeptide alkaloid & Ethanol, benzene extract & $\begin{array}{l}\text { Spermatogenic elements of testis and } \\
\text { epididymal sperm count., androgenic } \\
\text { activity }\end{array}$ & $\begin{array}{l}\text { Reddy et al. (1997); Gupta et al. } \\
\text { (1985) }\end{array}$ \\
\hline 23. & $\begin{array}{l}\text { Momordica charantia L. } \\
\text { Cucurbitaceae } \\
\text { Karwellaka } \\
\text { Roots and leaves }\end{array}$ & $\begin{array}{l}\text { Glycosides, saponins, alkaloids, fixed oils, } \\
\text { triterpenes, proteins, and steroids }\end{array}$ & Aqueous & $\begin{array}{l}\text { Antispermatogenic, antisteroidogenic } \\
\text { activity }\end{array}$ & Naseem et al. (1998) \\
\hline 24. & $\begin{array}{l}\text { Ocimum sanctum L. } \\
\text { Lamiaceae, Tulsi, leaves }\end{array}$ & $\begin{array}{l}\text { Carvacrol, sesquiterpene, hydrocarbon, and } \\
\text { caryophyllene }\end{array}$ & Benzene extract & $\begin{array}{l}\text { Decreased sperm count, weight of testis, } \\
\text { and sperm motility }\end{array}$ & Pandey and Madhuri (2010) \\
\hline
\end{tabular}


TABLE 2 | (Continued) Medicinal plants and their phyotoconstituents validated for various female/male contraceptive activities. Different contraceptive activities studied on medicinal plants could be categorized as follows. Female contraceptive activities: (2A) anti-implantation activity, (2B) abortification, (2C) antifertility, (2D) antiovulatory, and (2E) antiestrogenic activity. Male contraceptive activities: (2F) antispermatogenic, (2G) spermicidal, and $(2 \mathrm{H})$ antiandrogenic activity.

25. Piper betle L.

Piperaceae

Betel leaf, Pan

Petiole

26.

Piper nigrum L.

Piperaceae

Marich, Black pepper

Fruit

27.

Plumbago zeylanica L.

Plumbaginaceae

28.

Chitrak, Root

Fabaceae

Raktachandan

Pueraria tuberosa (Willd.) DC.

Fabaceae, Varahikand, rhizome

Semecarpus anacardium L.f.

Anacardiaceae

Bhallatak, Marking nut, Seeds

Wight \&Arn.

Combretaceae

Tylophora asthmatica (L. f.) Wight

\&Arn. Apocynaceae

Khadki Rasna

Chemical composition

Extract

Mode of action in experimental studies

Reference

G Spermicidal

activity

Acacia concinna (Willd.) DC

Leguminosae-Mimosoideae

Shikekai, stem bark

2. Achyranthes aspera L.

Amaranthaceae

ApamargaWhole plant,

Stem bark, Root

3. Alstonia scholaris (L.) R.Br

Apocynaceae

Saptaparna, stem bark

4. Azadirachta indica A. Juss.

Meliaceae

Nimba

Leaves, flower, and seed

5. Bambusa bambos (L.) Voss

Poaceae, Vansha

Tender stem
Eugenol, eugenol acetate, piper betol, piperol, Alcoholic extract methyl eugenol, and phytol

Piperine

Plumbagin

Santalin A, B, savinin, calocedrin, pterolinus $K, L, \quad$ Water and pterostilbenes

Puerarin, genistein, and daidzein

Methanol

Bhilwanols, phenolic compounds, biflavonoids, Ethanolic and sterols glycosides

Tannins, triterpenoid saponins, flavonoids, gallic Crude form acid, ellagic acid, and phytosterol

Aempferol, quercetin, tyloindane, cetyl-alcohol, tannins, glucose, calcium salts, and potassium chloride

Hexacosanol, spinasterone, oxalic, tartaric citric, succinic, ascorbic acid, alkaloids

calyctomine, and nicotine

Fatty acids, oleonic acid, bisdesmosidic

Alcoholic

triterpenoid achyranthine

Erythrodiol, uvaol, betulin, oleanolic acid ursolic Water extrac acid, and $\beta$-amyrin

Azadirachtin, nimbolinin, nimbin, nimbidin, nimbidol, sodium nimbinate, and gedunin

Aqueous and Alcoholic

Balarenone, barlerin, barlerinosideverbascoside, Ethanolic acetylbarlerin, and lupulinoside sterile distilled water containing milk powder

thnol

Benzene, ethanolic, and

chloroform
Reduced sperm motility

Fruit powder-suspended in

reversible after cessation of treatment

al. (1999)

Antispermatogenic

Purohit et al. (2008)

Semen coagulating activity

Dhawan et al. (1980)

nhibition of spermatogenesis

upta et al. (2004), Gupta et al. (2005b)

Reduction in the number of primary spermatocytes, secondary spermatocytes, (2003)

and spermatids

Inhibition of spermatogenesis

Jha and Dixit (1986), Lal and Udupa (1993)

Antispermatogenic activity

Dikshith et al. (1990)

Decline germ cell population

Spermicidal effect on number of spermatozoa and level of fructose

Reduced sperm motility (1997)

Raj et al. (2011); Shibeshi et al. 2006): Vasudeva and Sharma (2006)

Gupta et al. (2003), 2004)

Gediya et al. (2011), Kasturi et al.

Vanithakumar et al. (1989)

(Continued on following page) 
TABLE 2 | (Continued) Medicinal plants and their phyotoconstituents validated for various female/male contraceptive activities. Different contraceptive activities studied on medicinal plants could be categorized as follows. Female contraceptive activities: (2A) anti-implantation activity, (2B) abortification, (2C) antifertility, (2D) antiovulatory, and (2E) antiestrogenic activity. Male contraceptive activities: (2F) antispermatogenic, (2G) spermicidal, and $(2 \mathrm{H})$ antiandrogenic activity.

Botanical name, family,

Sanskrit name, parts

Chemical composition

Extract

Mode of action in experimental studies

Reference

Cannabis sativa L

Cannabinoids, terpenes, and sesquiterpenes

Butin

Testicular lesion

Dixit and Joshi (1982)

Bhanga, leaves

Clirullus colocynthis (L.) Schrad. Carbohydrate, protein, amino acid, tannins,

Ethanol

Impairment of sperm

Chaturvedi and Dixit (1997)

Cucurbitaceas

saponins, phenolics, and cardicglycoloids

Bitter apple, fruits

Apiaceae

Essential oil

Grinjanak, Seed

Embelia ribes Burm.t.

Embelin

Petroleum, ether, benzene, Spermicidal activity

alcohol, and water

Reversiblecontacepir

Embelin in 50 and $100^{\circ} \mathrm{mg} / \mathrm{kg}$

Reversible contraception like activity in male

Garg (1975); Jansen and

Wolhlmuth (2014); Shah and

Varute (1980)

13. Terminalia bellirica (Gaertn.)

14.

16.

(1983)

$H$ Antiandrogenic

activity

1.

Primulaceae

Mentha arevensis $\mathrm{L}$ Alkaloids, steroids, and glycosides

Alkaloids, steroids, and glycosides Petroleum ether

Lamiaceae

Myristicin, elemicin, myristic acid, alpha-pinene,

Ethanol dogs

terpenes, beta-pinene, and trimyristin

Myristicaceae

Nutmeg, Jatiphal, seeds

Strychnos potatorum L.f.

Strychnine

Seed extract

Spermicidal Decreased weight of testis, sperm motility, and viability

Premature ejaculation

suppressive effects on male fertility

Nirmali, Seeds

Phenolic acids, saponins, lignans, triterpenoids, resveratrol glycosides, arjungenin, $\beta$-sitosterol,

Roxb.

Combretaceae

Bibhitak

Fruits

Tinospora cordifolia (Willd.)

and stigmasterol

Berberine, palmatine $\mathrm{D}$, choline $\mathrm{D}$, diterpene,

Hook.t.

terpenoids alkaloids, and steroids

Aqueous

\& Thomson

Menispermacea

Amrita Giloe

Trigonella foenum-graecum L., $\quad$ Water, carbohydrates, protein, fat, and calcium Aqueous

Fabaceae

Methika, Seeds

Withania somnifera (L.) Dunal Withanolides

Stem, ethanolic

Spermicidal activity in rat semen, human

semen

Ashwagandha

Stem and root

Aloe barbadensis Mill.

Water, polysaccharides, pectin, cellulose,

Synonym of Aloe vera (L.) Burm.f. hemicellulose, and glucomannan

Sharma and Jacob (2001a)

Mishra and Shukla (1980)

Gupta et al. (2006)

Kaur et al. (2011)

Spermicidal Reduced weight of testis,

Gupta and Sharma (2003)

sperm count

Spermicidal activity in human and rat semen Priya et al. (2012)

Reversible spermicidal and infertilizing effect Singh et al. (2013); Mali (1999)

(aceae

Kumari, leaves 
TABLE 2 | (Continued) Medicinal plants and their phyotoconstituents validated for various female/male contraceptive activities. Different contraceptive activities studied on medicinal plants could be categorized as follows. Female contraceptive activities: (2A) anti-implantation activity, (2B) abortification, (2C) antifertility, (2D) antiovulatory, and (2E) antiestrogenic activity. Male contraceptive activities: (2F) antispermatogenic, (2G) spermicidal, and $(2 \mathrm{H})$ antiandrogenic activity.

\begin{tabular}{|c|c|c|c|c|c|}
\hline Sr. No. & $\begin{array}{l}\text { Botanical name, family, } \\
\text { Sanskrit name, parts }\end{array}$ & Chemical composition & Extract & Mode of action in experimental studies & Reference \\
\hline 2. & $\begin{array}{l}\text { Aristolochia indica L. } \\
\text { Aristolochiaceae } \\
\text { Ishwari, roots }\end{array}$ & $\begin{array}{l}\text { Aristolochic acid, ceryl alcohol, } \beta \text {-sitosterol, } \\
\text { stigmast-4-en-3-one, friedelin, and } \\
\text { cycloeucalenol }\end{array}$ & Aristolochic acid & Antiandrogenic effects on langur monkey & Gupta et al. (1996) \\
\hline 3. & $\begin{array}{l}\text { Andrographis paniculata } \\
\text { (Burm.f.) Nees } \\
\text { Acanthaceae } \\
\text { Kirattikta, leaves }\end{array}$ & $\begin{array}{l}\text { Andrographolide, andrographidoids A, B, C, D, } \\
\text { E, diterpenoid, and lactone }\end{array}$ & Water extract & Antiandrogenic & $\begin{array}{l}\text { Akbarsha et al. (1990); Akbarsha } \\
\text { and Murugaian (2000) }\end{array}$ \\
\hline 4. & $\begin{array}{l}\text { Azadirachta indica A. Juss., } \\
\text { Meliaceae } \\
\text { Nimba } \\
\text { Leaves, flower, and seed }\end{array}$ & $\begin{array}{l}\text { Azadirachtin, nimbolinin, nimbin, nimbidin, } \\
\text { nimbidol, sodium nimbinate, and gedunin }\end{array}$ & Seed oil & Antiandrogenic & $\begin{array}{l}\text { Sharma et al. (1987); Sinha et al. } \\
\text { (1984); Roop et al. (2005) }\end{array}$ \\
\hline 5. & $\begin{array}{l}\text { Cuscuta reflexa Roxb } \\
\text { Convolvulaceae } \\
\text { Amarwel, whole plants }\end{array}$ & Alkaloids & Methanolic & Antisteroidogenic & Gupta et al. (2003) \\
\hline 6. & $\begin{array}{l}\text { Curcuma longa L. } \\
\text { Zingiberaceae } \\
\text { Haldi, rhizome }\end{array}$ & Curcumin and flavanoids & Ethanol, aqueous & Antiandrogenic & Bhagat and Purohit (1986) \\
\hline 7. & $\begin{array}{l}\text { Foeniculum vulgare Mill } \\
\text { Apiaceae } \\
\text { Common fennel, seeds }\end{array}$ & $\begin{array}{l}\text { Anethole, alpha pinene, beta myrcene-pinene, } \\
\text { bitter fenchone, camphene, and estragole }\end{array}$ & Alcoholic & Antiandrogenic & Farooq et al. (1997) \\
\hline 8. & $\begin{array}{l}\text { Hibiscus rosa-sinensis L. } \\
\text { Malvaceae } \\
\text { Japa, Flowers }\end{array}$ & Cyclopeptide alkaloid & Ethanol and Benzene extract & $\begin{array}{l}\text { Spermatogenic elements of testis and } \\
\text { epididymal sperm count., androgenic } \\
\text { activity }\end{array}$ & $\begin{array}{l}\text { Reddy et al. (1997); Gupta et al. } \\
\text { (1985) }\end{array}$ \\
\hline 9. & $\begin{array}{l}\text { Mucuna urens (L.) Medik. } \\
\text { Fabaceae } \\
\text { Horase been, Kapikacchu } \\
\text { Seeds }\end{array}$ & $\begin{array}{l}\text { L-DOPA, with trace amounts of serotonin, } \\
\text { nicotine, and bufotenine }\end{array}$ & Water & Effect on gonads and sex accessory glands & Udoh and Ekpenyong (2001) \\
\hline 10. & $\begin{array}{l}\text { Nicotiana tabacum L. } \\
\text { Solanaceae } \\
\text { Tobacco, leaves }\end{array}$ & $\begin{array}{l}\text { Lipid constituents, free fatty acids, triglycerides, } \\
\text { and sterol esters free sterols }\end{array}$ & Nicotine & Antiandrogenic & Londonkar et al. (1998) \\
\hline 11. & $\begin{array}{l}\text { Plumbago zeylanica L. } \\
\text { Plumbaginaceae } \\
\text { Chitrak, root }\end{array}$ & Plumbagin & Plumbagin-free alcohol & Antiandrogenic & Bhargava (1984) \\
\hline 12. & $\begin{array}{l}\text { Ruta graveolens } L . \\
\text { Rutaceae, Rue, leaves }\end{array}$ & Volatile oil & Aqueous extracts & $\begin{array}{l}\text { Adverse effects on territorial aggression and } \\
\text { sexual behavior in male albino rats }\end{array}$ & Khouri and Akawi (2005) \\
\hline 13. & $\begin{array}{l}\text { Semecarpus anacardium L.f. } \\
\text { Anacardiaceae } \\
\text { Bhallatak, Marking nut, Seeds }\end{array}$ & $\begin{array}{l}\text { Bhilwanols, phenolic compounds, biflavonoids, } \\
\text { and sterols glycosides }\end{array}$ & Aqueous extracts & Antiandrogenic & Singh (1985) \\
\hline
\end{tabular}


a cost-effective herbal contraceptive for its spermicidal property and is considered safe for regular use. (Achintya, 2018).

\section{Ricinus communis $\mathbf{L}$.}

The seeds of Ricinus communis Linn RICOM-1013-J, administered as a single oral dose of $2.3-2.5^{\circ} \mathrm{g}$ once $/ 12^{\circ}$ months acted as protection against pregnancy in 50 women volunteers. The study revealed very minimal side effects. The antifertility and contraceptive efficacy of RICOM-1013-J is due to hormonal mechanisms (Isichei et al., 2000). Goncim et al. (2010) stated that one seed of Ricinus communis L. taken orally can prevent ovulation in humans and the anticonceptive effect may be due in part to the prevention of ovulation.

\section{Compound Formulation}

A study was conducted on a combination of Ashoka (Saraca indica L.), Vidanga (Embelia ribes Burm.f.), Laksha (lac), and Kramuk (Areca nut) on 834 young, healthy patients in active reproductive age below $40^{\circ}$ years. The drug was administered from the 5 th $^{\circ}$ day of LMP for a period of $15^{\circ}$ days in a daily dose schedule of $1^{\circ} \mathrm{gm}$ (2 tablets) at bedtime with milk. Results suggested that the failure rate of treatment $1.19 / \mathrm{HWY}$ is comparable to both steroidal oral contraceptive pills and intrauterine device. It does not affect the hypothalamopituitary axis and did not have any other adverse effects. It can be a good alternative for lactating women (Palep and Jukar, 2003).

Central Council for Research in Ayurveda and Siddha had taken up a number of studies to evaluate the efficacy of Ayurvedic formulations like K Capsule, Ayush AC-IV, Pippalyadi yoga (in three different doses), Ayush AC II, Talisadi yoga, Vidangadi yoga, etc., which were proved as safe and effective in different clinical studies. Besides this, the council also tried the efficacy of neem oil-as a local contraceptive and found encouraging results (Galib et al., 2008).

\section{TERATOGENIC EFFECT}

Ayurveda classical texts have references to congenital birth [anmabalapravrita] disorders as per the etiopathology and clinical presentation. Some congenital malformations in the fetus may occur but the mechanism is still not clear.

Teratogen is an agent or factor that causes malformation in the embryo. One of the causes of malformation may be toxic substances such as drugs and environmental toxins in pregnancy.

Herbal drugs with appropriate dose and duration may not cause teratogenic effect but in the case of excess dose with improper mode of administration, for a longer duration than therapeutically advised, teratogenic effect may be seen. Scientific validation of their safe use in pregnancy is hardly documented. Teratogenic effects of some of the medicinal plans have been mentioned in Table 3.

It is observed that drugs having contraceptive and abortifacient action have potent teratogenic effect in experimental models. There are several studies of teratogenicity on other herbal drugs which are not showing teratogenic effects in low doses and may cause teratogenic effects in high doses, for example, Ashwagandha (Withania somnifera (L.) Dunal), Punarnava (Boerhavia diffusa L.), Narangi (Citrus aurantium L), Nimba (Azadirachta indica A. Juss.), Jatamansi (Nardostachys jatamansi (D.Don) DC.), (Bala Abutilon indicum L.) Sweet), and Yastimadhu (Glycyrrhiza glabra L.) (Jati, 2018).

Different contraceptive activities in the abovementioned 94 plant ingredients are categorized in Table 4.

\section{DISCUSSION}

Presently, scientifically established methods of contraception and contraceptive drugs are used extensively. The synthetic contraceptive drugs known to interfere with the endocrine system and natural hormones may produce reproductive, neurological, developmental, and metabolic adverse effects that are serious at times. Search for safer drugs and preference for natural origin contraceptive drugs and methods are of research interests. Necessarily, the objectives for research of novel contraceptives from nature would be the assurance regarding effectiveness, safety, and user compliance. There are many plants known to have antifertility activity both in male and female. Some of these plants had spermicidal and altered hormone levels.

The classical Ayurvedic texts offer substantial knowledge on reproductive biology for healthy progeny and medieval Ayurvedic and specific Sanskrit texts provide information about methods and a broad range of therapeutics and ingredients that are described for use in contraception. These include local and oral contraceptives, abortifacients, and other methods of antifertility and birth control. These formulations and ingredients are a valuable source for extended research in the field of contraception.

In this study, 94 indigenous medicinal plants have been reviewed. Chemotaxonomically, it is of interest to note that the maximal number of plants having abortifacient and contraceptives are from Fabaceae, Acanthaceae, Euphorbiaceae, and Liliaceae families.

\section{Ingredients, Phytoconstituents, and Contraceptive Activities}

Certain alkaloids, glycosides, saponins, tannins, terpenoids, and other phytoconstituents are known to disrupt ovarian functions and estrous cyclicity through interplay of ovarian and extra ovarian hormones. Alkaloids are a major group of secondary metabolites bitter in taste that stimulate the central nervous system or directly work on the human brain. These are antiparasitic, antiplasmodial, anticorrosive, antioxidative, antibacterial, anti-HIV, and have insecticidal activities. In a review, it has been suggested that maximum alkaloids containing plant drugs have been reported to have an antifertility, antiovulatory, anti-implantation, abortifacient effect on animals (Choudhury and Jadhav, 2013).

A majority of these medicinal ingredients used either in formulations or singly over centuries have also been studied for a variety of pharmacological, biological, and therapeutic activities. 


\section{Achyranthes aspera L.}

A plant known to have antimicrobial, hypolipidemic, and has antifertility qualities is also used to treat asthma and cough.

\section{Fruits of Annona squamosa L.}

A known insecticidal, antiovulatory, and abortifacient plant that is hematinic, cooling, a sedative, stimulant, expectorant, and tonic. Its seeds are abortifacient and insecticidal and are used to destroy lice in the hair.

\section{Calotropis gigantea $\mathrm{L}$.}

Calotropis gigantea L. having certain antifertility glycosides and cardenolides is used for colic pain, flatulence, asthma, cough, and whooping cough and has wound healing, anticancer, and hypoglycaemic effects. Calotropis Madar rootbark is used for abortive purposes and in India is used as an antidote and in the treatment of elephantiasis, leprosy, and chronic eczema.

\section{Camphor}

Camphor, the well-known aromatic, has hormone-modulating, contraceptive, abortifacient, and lactation-inhibiting properties in women. It has a dose-dependent effect in human sperm motility and viability. Camphor can pass the placental barrier and affect embryo development. Camphorcontaining compounds have shown uterotrophicantitussive, anticonvulsant, nicotinic receptor blocking, anti-implantation, antiestrogenic, as well as estrogenic activities and can reduce serum triglyceride and thyroid hormone.

Flowers of Hibiscus rosa-sinensis L containing quercetin-7-Ogalactoside, polyphenolic compounds, and kaempferol, having antispermatogenic compounds, is prescribed for contraception

\section{TABLE 3 | List of drugs with teratogenic effect}

\begin{tabular}{|c|c|c|c|c|}
\hline $\begin{array}{l}\text { Sr. } \\
\text { No. }\end{array}$ & Name of plants & Phytoconstituent & Dose and duration & Teratogenic effect \\
\hline \multirow[t]{2}{*}{1} & $\begin{array}{l}\text { Asparagus racemosus } \\
\text { Willd. }\end{array}$ & Shatavarin, Racemosol & $\begin{array}{l}1000^{\circ} \mathrm{mg} / \mathrm{kg} / \mathrm{body} \text { weight for } 60 \text {-day Charles } \\
\text { foster rat pups }\end{array}$ & $\begin{array}{l}\text { Prenatal study-increased resorption of fetus, } \\
\text { gross malformation i.e., swelling in legs, IUGR } \\
\text { with small placental size. }\end{array}$ \\
\hline & Root & & Methanolic extract & $\begin{array}{l}\text { Postnatal study-decreased number of pups } \\
\text { per litter and increased mortality of pups and } \\
\text { delayed developmental parameters Goel et al. } \\
\text { (2006) }\end{array}$ \\
\hline 2 & $\begin{array}{l}\text { Datura metel L. } \\
\text { Leaves }\end{array}$ & Atropine alkaloids & $500^{\circ} \mathrm{mg} / \mathrm{body} \mathrm{kg}$ wt rats, ethanolic extract & $\begin{array}{l}\text { Teratogenic in the late stage of pregnancy Azeez } \\
\text { and Philip (2013) }\end{array}$ \\
\hline 3 & $\begin{array}{l}\text { Gloriosa superba L. } \\
\text { Tuber }\end{array}$ & Colchicine & $\begin{array}{l}1-3 \text { ppm and } 4-5 \text { ppm } \\
\text { Hydroalcoholic extract }\end{array}$ & $\begin{array}{l}\text { Antifertility activity scarcely produced abnormal } \\
\text { embryos. Induce high percentage of } \\
\text { abnormalities. Badwaik (2011) }\end{array}$ \\
\hline 4 & Lawsonia inermis L. & Flavonoid and phenolic compounds & $\begin{array}{l}100^{\circ} \mathrm{mg} / \mathrm{kg} \text { body wt. BALB/c mice between } 8- \\
12^{\circ} \mathrm{wk} \text { hydroalcoholic extract }\end{array}$ & $\begin{array}{l}90 \% \text { embryo, more extra ribs anencephaly, } \\
\text { exencephaly, skeletal abnormalities, height and } \\
\text { weight loss in embryos Lobat (2015) }\end{array}$ \\
\hline 5 & $\begin{array}{l}\text { Luffa operculata (L.) } \\
\text { Cogn. } \\
\text { Tea, decoction }\end{array}$ & $\begin{array}{l}\text { Glycosides, saponins, resin, free } \\
\text { sterols, aliphatic esters, quinones }\end{array}$ & $\begin{array}{l}\text { After ingestion of a variable amount of tea made } \\
\text { with dried fruit, decoction }\end{array}$ & Abortion, reduction in birth rate Barilli et al. (2005) \\
\hline 6 & Plumbago zeylanica L & Plumbagin & $\begin{array}{l}100^{\circ} \mathrm{mg} / \mathrm{body} \mathrm{kg} \text { wt orally with } 0.5^{\circ} \mathrm{ml} \text { of distilled } \\
\text { water in mice }\end{array}$ & $\begin{array}{l}\text { Stunted growth, subcutaneous, and deep } \\
\text { hemorrhage, kinking of tail, protrusion of back of } \\
\text { head Srivastava (2017) }\end{array}$ \\
\hline 7 & Ruta graveolens L. & Essential oil & $\begin{array}{l}5,10 \text {, and } 20 \% \text { w/v or plain water (control) orally } \\
\text { for } 4 \text { days }\end{array}$ & $\begin{array}{l}\text { Changes in the blastocyst formation, reducing } \\
\text { the number, and delaying the development of } \\
\text { embryos Gutiérrez-Pajares et al. (2003) } \\
\text { embryotoxic effect De Freitas et al. (2005) }\end{array}$ \\
\hline 8 & $\begin{array}{l}\text { Sena (Senna) } \\
\text { alexandrina Mill- } \\
\text { Fabaceae }\end{array}$ & Sennosides & Extract & $\begin{array}{l}\text { Increase blood flow to the uterus and its } \\
\text { attachments, increasing the risk of fetal loss, and } \\
\text { may pass spasms in the infant } \\
\text { Schulz et al. (2002) }\end{array}$ \\
\hline 9 & $\begin{array}{l}\text { Zingiber officinale } \\
\text { Roscoe }\end{array}$ & $\begin{array}{l}\text { Carbohydrates (50-70\%), lipids } \\
\text { (3-8\%), terpenes, and phenolic } \\
\text { compounds }\end{array}$ & $\begin{array}{l}\text { Orally at } 0,250,500,1000 \text {, or } 2000^{\circ} \mathrm{mg} / \mathrm{kgbw} / \\
\text { day-five groups }\end{array}$ & $\begin{array}{l}\text { High dose significantly reduced the number of } \\
\text { live fetuses, increased fatal death, and } \\
\text { resorption. Reda et al. (2018) }\end{array}$ \\
\hline 10 & Pipalyadi gutika & Piperine & $\begin{array}{l}5 \text { times to one and five times to the other than } \\
\text { the recommended dose for humans Rats }\end{array}$ & $\begin{array}{l}\text { Fetus - LBW, smaller in length, developmental } \\
\text { defects of soft tissues, skeletons, herniation of } \\
\text { intestines into umbilical cord, } \\
\text { Mother-less weight gain during gestation } \\
\text { Chaudhury et al. (2001) }\end{array}$ \\
\hline 11 & $\begin{array}{l}\text { Vishamustivati } M] \text { \& } \\
\text { Shuddha Tankana [ST] }\end{array}$ & - & $\begin{array}{l}175^{\circ} \mathrm{mg} / \mathrm{kg} \text { of aqueous solutions of } \\
\text { VisamustiVati, } 300^{\circ} \mathrm{mg} / \mathrm{kg} \text { aqueous solutions of } \\
\text { SuddhaTankana, orally from day } 1 \text { to day } 7 \text { of } \\
\text { post mating period }\end{array}$ & $\begin{array}{l}\text { W and ST shows positive Teratological effect on } \\
\text { new-borns, gross remarkable external } \\
\text { morphological and skeletal defects Jati (2018) }\end{array}$ \\
\hline
\end{tabular}


TABLE 4 | List of medicinal plants with one or more contraceptive activities.

\begin{tabular}{|c|c|c|c|c|c|c|c|c|c|}
\hline Sr. No & $\begin{array}{l}\text { Plant } \\
\text { name }\end{array}$ & $\begin{array}{l}\text { Anti- } \\
\text { implantation }\end{array}$ & Abortification & Antifertility & Antiovulatory & $\begin{array}{l}\text { Antiestrogenic } \\
\text { activity }\end{array}$ & Antispermatogenic & Spermicidal & $\begin{array}{l}\text { Antiandrogenic } \\
\text { activity }\end{array}$ \\
\hline 1 & Abies spectabilis (D.Don) Mirb. & $\sqrt{ }$ & & & & & & & \\
\hline 2 & Abroma augusta (L.) L.f. & $\sqrt{ }$ & $\sqrt{ }$ & & & & & & \\
\hline 3 & Abrus precatorius $\mathrm{L}$. & & $\sqrt{ }$ & $\sqrt{ }$ & & & $\sqrt{ }$ & & \\
\hline 4 & Acacia concinna (Willd.) DC. & & & & & & & $\sqrt{ }$ & \\
\hline 5 & Acacia leucophloea (Roxb.) Willd. & & & $\sqrt{ }$ & & & & & \\
\hline 6 & Achyranthes aspera $\mathrm{L}$. & & $\sqrt{ }$ & & $\sqrt{ }$ & & & $\sqrt{ }$ & \\
\hline 7 & Adhatoda vasica Nees & $\sqrt{ }$ & $\sqrt{ }$ & & & & & & \\
\hline 8 & Aegle marmelos (L.) Corrêa. & & $\sqrt{ }$ & & & & $\sqrt{ }$ & $\sqrt{ }$ & \\
\hline 9 & Ailanthus excelsa Roxb & $\sqrt{ }$ & & & & & & & \\
\hline 10 & Albizia lebbeck (L.) Benth. & & & & & & $\sqrt{ }$ & & \\
\hline 11 & Allium cepa $L$. & $\sqrt{ }$ & & & & & & & \\
\hline 12 & Allium sativum $\mathrm{L}$. & & & & & $\sqrt{ }$ & & & \\
\hline 13 & $\begin{array}{l}\text { Aloe barbadensis Mill. } \\
\text { Synonym of Aloe vera (L.) Burm.f. }\end{array}$ & $\sqrt{ }$ & & & & & & & $\sqrt{ }$ \\
\hline 14 & Alstonia scholaris (L.) R.Br. & & & & & & $\sqrt{ }$ & & $\sqrt{ }$ \\
\hline 15 & Andrographis paniculata (Burm.f.) Nees & & & & & & $\sqrt{ }$ & & $\sqrt{ }$ \\
\hline 16 & Ananas comosus (L.) Mer & & & & & & & $\sqrt{ }$ & \\
\hline 17 & Annona squamosa $\mathrm{L}$. & & $\sqrt{ }$ & & & & $\sqrt{ }$ & & \\
\hline 18 & Areca catechu L. & $\sqrt{ }$ & $\sqrt{ }$ & $\sqrt{ }$ & $\sqrt{ }$ & & $\sqrt{ }$ & & \\
\hline 19 & Aristolochia indica L. & & & & & & $\sqrt{ }$ & $\sqrt{ }$ & \\
\hline 20 & Azadirachta indica A. Juss. & & & $\sqrt{ }$ & $\sqrt{ }$ & & $\sqrt{ }$ & $\sqrt{ }$ & $\sqrt{ }$ \\
\hline 21 & Bacopa monnieri (L.) Wettst. & & & & & & $\sqrt{ }$ & & \\
\hline 22 & Balanites roxburghii Planch. & & & & & & $\sqrt{ }$ & & \\
\hline 23 & Bambusa bambos (L.) Voss & & & & & & & $\sqrt{ }$ & \\
\hline 24 & Barleria prionitis $L$. & & $\sqrt{ }$ & & & & & & \\
\hline 25 & Berberis aristata DC & & & & & & $\sqrt{ }$ & & \\
\hline 26 & Butea monosperma (Lam.) Kuntze & & & & $\sqrt{ }$ & & $\sqrt{ }$ & & \\
\hline 27 & Calotropis procera (Aiton) Dryand. & & & & $\sqrt{ }$ & & $\sqrt{ }$ & & \\
\hline 28 & Cannabis sativa $\mathrm{L}$. & & & & & & & $\sqrt{ }$ & \\
\hline 29 & Carica papaya L. & $\sqrt{ }$ & $\sqrt{ }$ & $\sqrt{ }$ & & & $\sqrt{ }$ & & \\
\hline 30 & Cassia fistula $\mathrm{L}$. & $\sqrt{ }$ & & & & & & & \\
\hline 31 & Catunaregam spinosa (Thunb.) Tirveng. & & & & & & & & \\
\hline 32 & Celastrus paniculatus Willd. & & & & & & $\sqrt{ }$ & & \\
\hline 33 & Centratherum anthelminticum (L.) Gamble & $\sqrt{ }$ & & & & & & & \\
\hline 34 & Cichorium intybus $L$. & & & & & & $\sqrt{ }$ & & \\
\hline 35 & Cinnamomum camphora (L.) J. Presl & & & & & & $\sqrt{ }$ & & \\
\hline 36 & Cissampelos pareira $\mathrm{L}$. & & & $\sqrt{ }$ & & & & & \\
\hline 37 & Citrullus colocynthis (L.) Schrad. & & & & & & & $\sqrt{ }$ & \\
\hline 38 & Citrus $\times$ aurantium $L$ & $\sqrt{ }$ & $\sqrt{ }$ & & $\sqrt{ }$ & & & & \\
\hline 39 & Crateva nurvala Buch. -Ham & & & $\sqrt{ }$ & & & & & \\
\hline 40 & Cuminum cyminum $L$. & & & $\sqrt{ }$ & & & $\sqrt{ }$ & & \\
\hline 41 & Cuscuta reflexa Roxb & & & & & & & & $\sqrt{ }$ \\
\hline 42 & Curcuma longa $\mathrm{L}$. & & & $\sqrt{ }$ & & & & & $\sqrt{ }$ \\
\hline 43 & Cyperus rotundus $L$. & & & & & $\sqrt{ }$ & & & \\
\hline 44 & Daucus carota $L$ & & $\sqrt{ }$ & $\sqrt{ }$ & & & & $\sqrt{ }$ & \\
\hline 45 & Desmodium gangeticum (L.) DC. & & & $\sqrt{ }$ & & & & & \\
\hline 46 & Embelia ribes Burm.f. & $\sqrt{ }$ & $\sqrt{ }$ & & & & $\sqrt{ }$ & $\sqrt{ }$ & \\
\hline 47 & Euphorbia neriifolia L. & & & & & & $\sqrt{ }$ & (Continued o & following page) \\
\hline
\end{tabular}


TABLE 4 | (Continued) List of medicinal plants with one or more contraceptive activities.

\begin{tabular}{|c|c|c|c|c|c|c|c|c|c|}
\hline Sr. No & $\begin{array}{l}\text { Plant } \\
\text { name }\end{array}$ & $\begin{array}{l}\text { Anti- } \\
\text { implantation }\end{array}$ & Abortification & Antifertility & Antiovulatory & $\begin{array}{l}\text { Antiestrogenic } \\
\text { activity }\end{array}$ & Antispermatogenic & Spermicidal & $\begin{array}{l}\text { Antiandrogenic } \\
\text { activity }\end{array}$ \\
\hline 48 & Ferula jaeschkeana Vatke & & & $\sqrt{ }$ & & & & & \\
\hline 49 & Foeniculum vulgare Mill & & & & & & & & $\sqrt{ }$ \\
\hline 50 & Gloriosa superba L. & $\sqrt{ }$ & $\sqrt{ }$ & $\sqrt{ }$ & & & & & \\
\hline 51 & Glycyrrhiza glabra L & & & & & $\sqrt{ }$ & & & \\
\hline 52 & Grewia asiatica L & $\sqrt{ }$ & $\sqrt{ }$ & & & & & & \\
\hline 53 & $\begin{array}{l}\text { Guilandina bonduc L. } \\
\text { Sy. Caesalpinia bonducella (L.) Fleming }\end{array}$ & & & & & $\sqrt{ }$ & & & \\
\hline 54 & Hibiscus rosa-sinensis L. & $\sqrt{ }$ & & $\sqrt{ }$ & $\sqrt{ }$ & & $\sqrt{ }$ & & $\sqrt{ }$ \\
\hline 55 & Lawsonia inermis $\mathrm{L}$. & & & $\sqrt{ }$ & & & & & \\
\hline 56 & Lepidium sativum $\mathrm{L}$ & & $\sqrt{ }$ & $\sqrt{ }$ & & & & & \\
\hline 57 & Melia azedarach L & & & $\sqrt{ }$ & & & & & \\
\hline 58 & Mentha arevensis $L$ & & & & & & & $\sqrt{ }$ & \\
\hline 59 & Mesua ferrea L. & $\sqrt{ }$ & & & & & & & \\
\hline 60 & Michelia champaca L. & $\sqrt{ }$ & & & & & & & \\
\hline 61 & Momordica charantia L. & $\sqrt{ }$ & & $\sqrt{ }$ & & & $\sqrt{ }$ & & \\
\hline 62 & Mucuna urens (L.) Medik & & & & & & & & $\sqrt{ }$ \\
\hline 63 & Musa paradisiaca L. & & & & $\sqrt{ }$ & & & & \\
\hline 64 & Myristica fragrans Houtt & & & & & & & $\sqrt{ }$ & \\
\hline 65 & Nelumbo nucifera Gaertn. & & & & & $\sqrt{ }$ & & & \\
\hline 66 & Nicotiana tabacum L & & & & & & & & $\sqrt{ }$ \\
\hline 67 & Nigella sativa L. & & & $\sqrt{ }$ & & & & & \\
\hline 68 & Ocimum sanctum L. & & & & & & $\sqrt{ }$ & & \\
\hline 69 & Papaver somniferum $\mathrm{L}$. & & & & $\sqrt{ }$ & & & & \\
\hline 70 & Piper betle L. & & & $\sqrt{ }$ & & & $\sqrt{ }$ & & \\
\hline 71 & Piper longum L. & & & $\sqrt{ }$ & & & $\sqrt{ }$ & & \\
\hline 72 & Piper nigrum L. & & & $\sqrt{ }$ & & & $\sqrt{ }$ & & \\
\hline 73 & Plumbago rosea $L$. & & & & $\sqrt{ }$ & & & & \\
\hline 74 & Plumbago zeylanica L. & $\sqrt{ }$ & & & & & $\sqrt{ }$ & & $\sqrt{ }$ \\
\hline 75 & Pterocarpus santalinus L.f. & & & & & & $\sqrt{ }$ & & \\
\hline 76 & Pueraria tuberosa (Willd.) DC & & & & & & $\sqrt{ }$ & & \\
\hline 77 & Ricinus communis $\mathrm{L}$. & $\sqrt{ }$ & $\sqrt{ }$ & & & & & & \\
\hline 78 & Ruta graveolens L & & & & & & & & $\sqrt{ }$ \\
\hline 79 & Sapindus trifoliatus L. & $\sqrt{ }$ & & & & & & & \\
\hline 80 & Semecarpus anacardium L.f. & & & & $\sqrt{ }$ & & $\sqrt{ }$ & & $\sqrt{ }$ \\
\hline 81 & Sesbania sesban (L.) Merr & $\sqrt{ }$ & & & & & & & \\
\hline 82 & Sesamum indicum L & & & & & $\sqrt{ }$ & & & \\
\hline 83 & Strychnospotatorum L.f. & & & & & & & $\sqrt{ }$ & \\
\hline 84 & Taxus baccata L & & & & $\sqrt{ }$ & & & & \\
\hline 85 & Terminalia arjuna (Roxb. ex DC.) Wight \&Arn & & & & & & $\sqrt{ }$ & & \\
\hline 86 & Terminalia bellirica (Gaertn.) Roxb & & & & & & & $\sqrt{ }$ & \\
\hline 87 & Tinospora cordifolia (Willd.) Hook.f.\& Thomson & & & & & & & $\sqrt{ }$ & \\
\hline 88 & Trichosanthes cucumerina L. & & & $\sqrt{ }$ & & & & & \\
\hline 89 & Trigonella foenum-graecum L. & & & & & & & $\sqrt{ }$ & \\
\hline 90 & Tylophora asthmatica (L. f.) Wight \& Arn & & & & & & $\sqrt{ }$ & & \\
\hline 91 & Vitex negundo L. & & & & $\sqrt{ }$ & $\sqrt{ }$ & & & \\
\hline 92 & Withania somnifera (L.) Dunal & & & & & & & $\sqrt{ }$ & \\
\hline 93 & Woodfordia fruticosa (L.) Kurz & & $\sqrt{ }$ & & & & & & \\
\hline 94 & Zingiber officinale Roscoe & & & $\sqrt{ }$ & & & & & \\
\hline
\end{tabular}




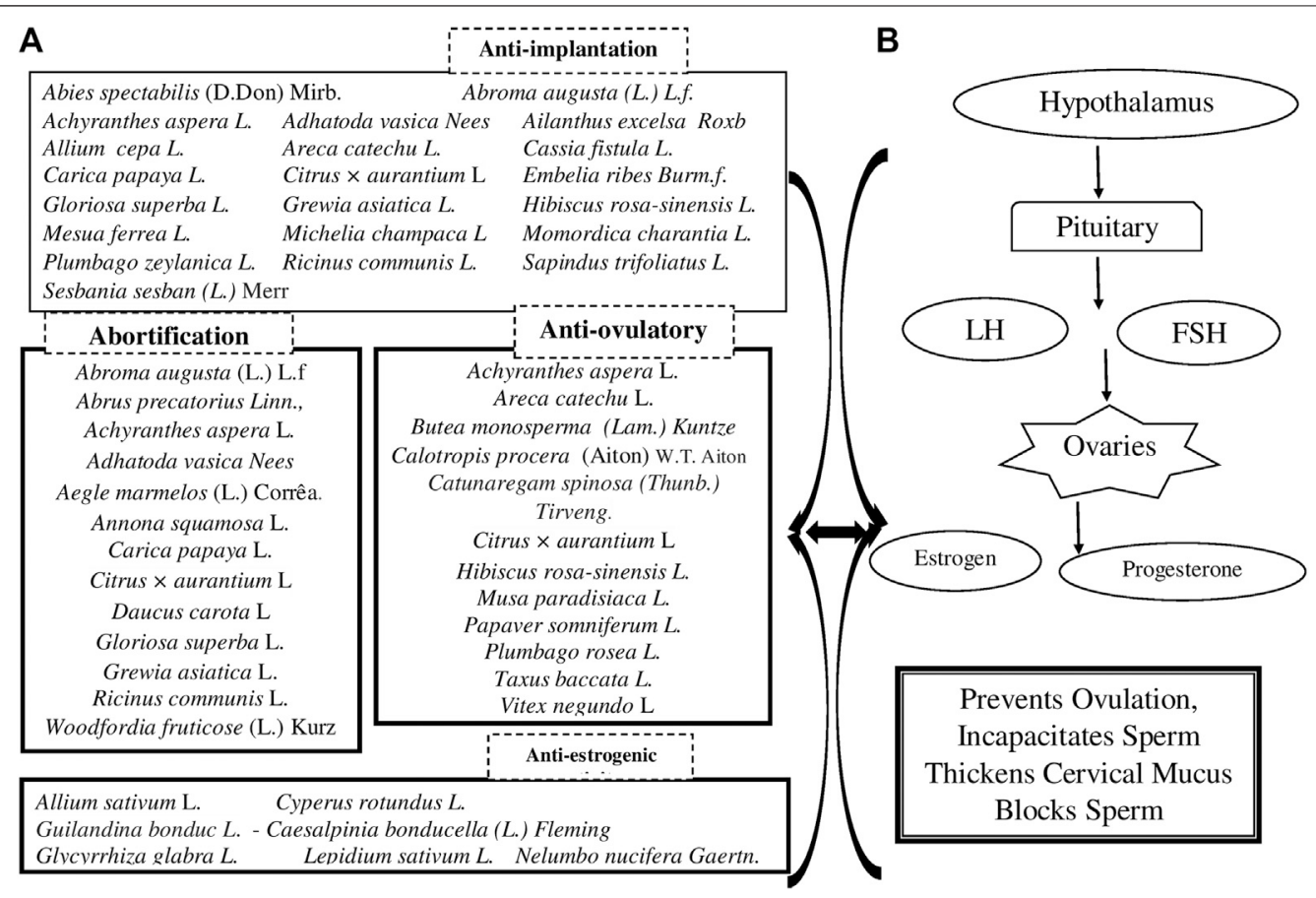

FIGURE 4 | Female contraceptive plants and possible mechanism.

and is used to treat bacterial infection, hyperlipidemia, and depression and act as an antioxidant.

Two of the most bitter stimulant plants, Momordica charantia L. and Azadirachta indica A. Juss., produce an irregular pattern of estrous cycle with prolonged diestrus phase. Steroids, triterpenoids, reducing sugars, alkaloids, phenolic compounds, flavonoids, and tannins in the plant cause reduction in the number of normal follicles because of atresia which occur due to disruption of the process of follicle selection. Azadirachta arrests spermatogenesis and androgen depletion.

Roots of Plumbago zeylanicum L. have been used as an abortifacient, internally or as an irritant to the uterus. This acrid and stimulant root increases appetite helps indigestion and is used for dyspepsia, piles, and skin diseases. It induces sweating, its powder is occasionally taken as snuff to relieve headache, and it helps in the adhesion of tissues in the body and is antidiarrheal.

Tinospora cordifolia (Willd.) Hook.f. and Thomson, an immunomodulator plant used to treat tuberculosis, fever, and wounds, has antifertility qualities. It is used for antioxidant, hypoglycaemic, and cardioprotective activities.

Excessive use of substances having pungent, bitter, and astringent tastes is contraindicated for sexual functions. Excess consumption of bitter taste leads to loss of strength and energy, astringent taste affects the sperm count, and can even reduce the sex drive while strongly pungent ingredients like pepper exhibit spermicidal or abortifacients effects.
Prolonged consumption of these tastes may lead to emaciation of the body.

\section{Mechanism of Action Female Contraceptives}

Medicinal plants may induce infertility in distinct ways. They may affect the ovarian, uterine, and hormone production functions and interfere with implantation or sperm production. These drugs are of natural origin, hydrophilic, and lipophilic; can traverse paracellularly through the vaginal mucosa; and exhibit its efficacy as contraceptive, by altering the vaginal $\mathrm{pH}$. These drugs may variably act locally to bring changes in the cervical mucus and alter decidual embedding and thereby act as anti-implantation agents, or may inhibit propulsion of sperm in the fallopian tubes by altering tubal mechanism or may act on hormones as antiovulation agents. They may act through rapid expulsion of the fertilized ova from the fallopian tube or inhibit implantation due to disturbance of the estrogen progesterone balance or induce fetal abortion by inhibition of nutrition to the uterus and the embryo.

Moreover, plants with estrogenic property can directly influence pituitary action by peripheral modulation of luteinizing hormone (LH) and follicle stimulating hormone (FSH), decreasing their secretions and blocking ovulation (Brinker, 1997). Plants with antiestrogenic activities intercept in the process of development of ovum and endometrium and on the other hand, plants have abortifacient effects (Gark et al., 1978; Prakash et al., 1985). 

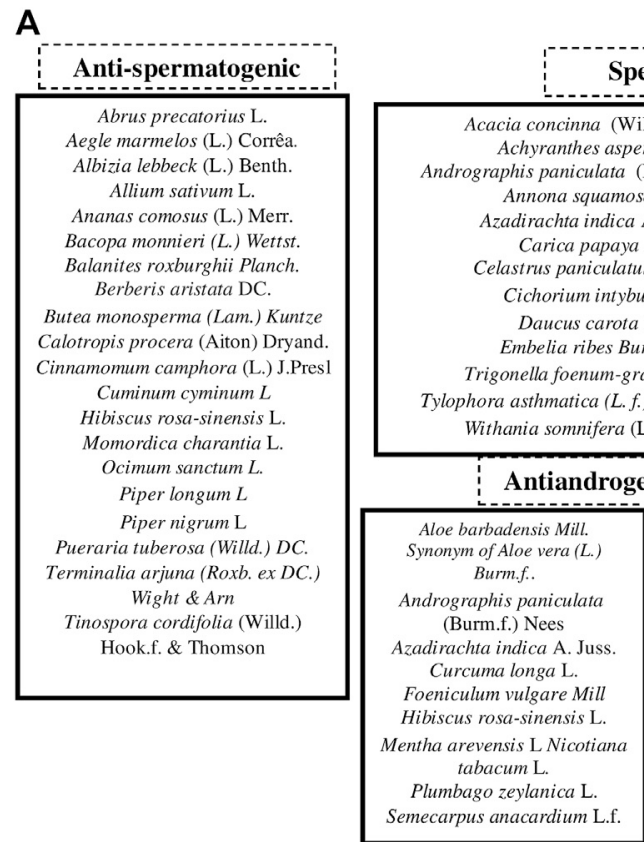

B

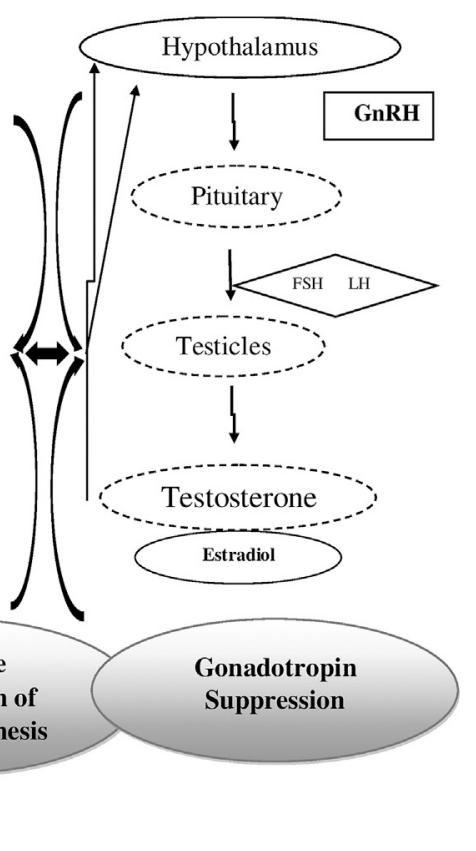

FIGURE 5 | Male contraceptive plants and possible mechanism.

The site of action of antifertility agents in females comprises the hypothalamus, the anterior pituitary, the ovary, the oviduct, the uterus, and the vagina. The mammalian uterus is the main site of antifertility effects (Williamson et al., 1996). Typical estrogenic compounds posses the ability to increase the uterine wet weight and induce cornification and opening of vagina in immature rats, which results in anti-implantation effects (Turner, 1971).

Antifertility plants prevent fertilization; these drugs obstruct the formation of gametes and interfere with the process of fertilization. Antiovulatory plants induce infertility by suppressing ovulation. Anti-implantation plants prevent the attachment or penetration of fertilized ovum into the uterus. Butea monosperma (Lam.) Kuntze, Ocimum sanctum L., Calotropis procera (Aiton) W.T. Aiton, Mentha arvensis, and Lawsonia inermis $L$-all have antiimplantation activity. Abortifacient plants cause early expulsion of the fetus. These act during the first five weeks of pregnancy as they block the action of progesterone so that the uterus sloughs off the embryo. Abrus precatorius L., Annona squamosa L., Calotropis procera (Aiton) W.T. Aiton, Carica papaya L., Dhatura metel L., Momordica charantia L., and Catunaregam spinosa (Thunb.) Tirveng are medicinal plant drugs which can be used as abortifacients. Stimulant, irritant, and bulk forming characteristics of these drugs facilitate abortion along with hormonal regulation and modulation of genital functioning. These ingredients are considered stimulants and are hot in nature and hence should be used for a short duration.

It observed that large numbers of antifertility plant extracts are known to exhibit estrogenic activity in rats (Dahanukar et al., 2000). Estrogenic substance may cause the expulsion of ova from the tube, disruption of luteotrophic activity of the blastocyst, and disrupt the functional equilibrium between the endogenous estrogen and progesterone, which may result in failure in fertility. Increase in the wet weight of uterus of substancetreated ovariectomized immature rats may indicate that the substance has an estrogenic effect (Mukherjee, 2002).

The hypothalamus has threshold requirement for estrogen to cause a massive release of LH by the pituitary gland. This surge of $\mathrm{LH}$ is the trigger, which initiates the rupture of the follicle (ovulation) (Bullock et al., 1995). It is known that an increase in the serum progesterone level prevents pregnancy through inhibition of ovulation and alteration of cervical mucus.

Most of the plants possess inhibition of implantation or reduction of estrogen level and increment of progesterone level as the possible mechanism of antifertility effect.

The anti-implantation effect may be due to the disturbance of endocrine-endometrial synchrony that is dependent on estrogen and progesterone balance. Factors other than the hormones such as histamine, prostaglandins, proteolytic enzyme NOS, alkaline phosphatase, interleukins, and leukemia-inhibitory factors, which are important for implantation, may also be affected by the various plant extracts (Gupta, 1994; Garg et al., 1978; Novaro et al., 1997; Prakash et al., 1989; Dimitriadis et al., 2003; Yang et al., 1994)

\section{Male Contraceptives}

Male contraceptive drugs may inhibit spermatogenesis or act on male hormones when used orally or may be spermistatic or spermicidal when used intravaginally. Male contraceptives might work to suppress sperm production by antispermatogenic or prevent maturation of sperm or prevent the flow of sperm through the vas deferens or deposition of the sperm (Soni et al., 2015). 
Plant extracts have also shown promising antifertility effects when administered to male rats. The various effects on male reproductive system to induce antifertility action shown by plants includes antispermatogenic effect, post-testicular antifertility effect, spermicidal effect, sperm-immobilizing effect, antiandrogenic effect, etc.

Antispermatogenic activity indicates interference in the steroidogenesis when the cholesterol level rises and sudanophilic lipid accumulates (Mandal et al., 2010). Some of the plant extracts kill the viability and work on Sertoli cells and have various effects on spermatogenesis, such as reducing the nuclear and cytoplasmic volume and vacuolizing Sertoli cells (Sharma RS et al., 2001) or acts through Leydig cells (Dufau et al., 1984). Some plant extracts act by unbalancing the hormones or through their antimotility activity (Verma and Yadav, 2021).

Spermicidals are contraceptive substances that destroy the sperm when inserted vaginally prior to intercourse. The spermicidal agents consist of a surfactant that destroys the sperm cell membrane. Lipid peroxidation may play an important role in disrupting the sperm membrane physiology that may or may not be accompanied with a detrimental effect on the defense system of the human spermatozoa against the ROS.

Antiandrogens, also known as androgen antagonists or testosterone blockers, prevent androgens like testosterone and dihydrotestosterone (DHT) from mediating their biological effects in the body. Andrographis paniculata (Burm.f.) Nees, Azadirachta indica A. Juss., Curcuma longa L., Hibiscus rosasinensis L., and Plumbago zeylanica L. act by blocking the androgen receptor (AR) and/or inhibiting or suppressing androgen production. They can be considered as the functional opposites of AR agonists, for instance, androgens and anabolic steroids (AAS) like testosterone, DHT, and nandrolone and selective androgen receptor modulators (SARMs) like enobosarm.

Figures 4, 5 provide group of these plants 3 (a) and 4 (a) with probable female and male contraceptive activities 3 (b) and 4 (b), respectively.

\section{Limitations/Challenges}

A major limitation is the contradictory reports or nonreproducibility of published data, which can provide useful leads. At times, failure of reproducibility of contraceptive activity of a plant or its constituent is observed. This could be due to the multiple factors at different levels that are known to affect the reproductive process. The other reason could be the variable effect of the herbal contraceptive/s in animals as against when used in humans.

The contraceptives of natural origin are not used much in practice, the main factor being the lack of standardization and reliable validation studies. The information has thus remained fragmented. Studies have consequently been scarce. Interest has weaned due to the complexity and enormity of the large and long-term study requirements covering multiple variables.

Analytical methods, information on phytoconstituents, availability of markers, and their activities have now provided new standardization approaches to herbal products that assure higher safety and stability.

The solution to this is to investigate the efficacy of these herbs in humans themselves, after ascertaining their safety in animal models. There is also a need to record the conditions under which the plants are used by indigenous people, including the time and place of collection, proper botanical authentication, and schedule of administration. Advances in biology offer adaptable and promising experimental models to examine the effectiveness of natural products for altering reproductive functions and contraception

\section{CONTRACEPTION AND NEW TECHNOLOGIES FOR NATURAL PRODUCTS}

There is a need to use new contraceptive methods to minimize the side effects. The following technological advances are relevant in the context of this review for discovery and development of novel contraceptives of natural origin.

o Ayurveda recommends fumigation as a method and as a therapeutic procedure to treat various diseases, including microbial infections. Ayurvedic methods of sterilization with fumigation can be alternated as a modern contraceptive with the help of nanotechnology. Natural novel bioactive compound drugs could be developed with novel drug-delivery systems.

o A team in the University of Washington has developed an electrically spun cloth with nanometer-sized fibers that get dissolved to release drugs, thus providing a platform for cheap, discrete, and reversible protection ("Drug-Eluting Fibers for HIV-1 Inhibition and Contraception").

o Pharmacy on a chip is one of the most exciting parts of the drug-delivery system. It is a chip implanted into the body which releases drugs at set intervals. It would release the hormones estrogen and progestogen over a specific period to stop the release of eggs from the ovaries and thus prevent pregnancy.

o Nanotechnology-based condom systems have the potential to prevent the spread of HIV and STIs.

o Transdermal drug delivery (TDD) is an alternative method of drug administration for drugs whose delivery by conventional oral, topical, intravenous, and intramuscular methods is of limited efficacy. Recent advances in TDD involve the use of nanoparticles (NPs), which exhibit great potential to enhance drug permeation across the skin.

o Skin patches containing microneedles is a painless and minimally invasive method of TDD in which micron-sized pores are created in the epidermis to allow delivery of drugs to the blood vessels present in the dermal layer of the skin.

o Researchers report on a technique for administering contraceptive hormones through special backings on jewelry such as earrings, wristwatches, rings, or necklaces. The contraceptive hormones are contained in patches applied to portions of the jewelry in contact with the skin, allowing the drugs to be absorbed into the body (Georgia Institute of Technology, 2019). 


\section{Possibilities for new means of drug development}

- Developing newer biotechnology-based cellular or molecular models that could better replicate reproductive processes.

- Methods that act after ovulation and interfere with sperm delivery or function in the male or in the female genital tract or both ought to be adopted.

- Design of nonhormonal contraceptive agents-as an alternative option to hormonal formulations-with the help of herbals.

- New delivery mechanisms that can act both short and long term; the possibilities are to develop herbal pessary, jelly, patches, and condoms, or mechanical devices with natural ingredients to optimize the effects.

- Methods which limit the side effects associated with systemic exposure should be developed in lower dosage forms to ensure efficacy.

- Technologies that markedly improve the cost, acceptability, and deliverability of contraceptives.

- Personalized contraception-human genome could minimize the side effects while maximizing health benefits at the individual level.

\section{CONCLUSION}

Fertility and contraception are continued subjects of biomedical research and innovation. Alternatives to unmet needs for safer contraception methods and drugs are searched for. Many Ayurvedic medicinal ingredients and compound formulations are claimed to inhibit male and female fertility as mentioned in the classical literature. Several of these validated drugs possess

\section{REFERENCES}

Abdelgader, A., and Elsheikh, A. (2018). Antiandrogenic Activity of Calotropis Procera Latex in Rats. Asian Pac. J. Reprod. 7, 129-135. doi:10.4103/2305-0500.233574

Achintya, M. (2018). Evaluation of Contraceptive Properties of Neem Oil - A Prospective Study. Sci. Cult. 84 (1-2), 67-70.

Adhikary, P., Banerji, J., Chowdhury, D., Das, A. K., Deb, C. C., Mukherjee, S. R., et al. (1989). Antifertility Effect of Piper Betle Linn. Extract on Ovary and Testis of Albino Rats. Indian J. Exp. Biol. 27, 868-870.

Agarwal, M., and Dixit, V. P. (1982). Effect of Balanites Roxburghiion Male Reproductive Tract of Langur Monkey. Allahabad: 52nd Annual Session of National Academy Science, 56.

Ahmad, S., Jamal, Y., and Mannan, A. (2011). Review of Some Medicinal Plants with Anti-fertility Activities. Unani Res. 1 (2), 24-28. doi:10.5530/ur.2.2011.6

Akbarsha, M. A., Manivannan, B., Hamid, K. S., and Vijayan, B. (1990). Antifertility Effect of Andrographis Paniculata (Nees) in Male Albino Rat. Indian J. Exp. Biol. 28 (5), 421-426.

Akbarsha, M. A., and Murugaian, P. (2000). Aspects of the Male Reproductive Toxicity/male Antifertility Property of Andrographolide in Albino Rats: Effect on the Testis and the Cauda Epididymidal Spermatozoa. Phytother. Res. 14 (6), 432-435. doi:10.1002/1099-1573(200009)14:6<432::aid-ptr622>3.0.co;2-i

Anonymous (1996). Pharmacological Investigations of Certain Medicinal Plants and Compound Formulations Used in Ayurveda and Siddh. New Delhi: Central Council of Research in Ayurved and Siddha, 474.

Ave Olivia, R., Purwakanthi, A., and Dewi, H. (2020). Antifertility Effect of Betel Nut (Areca Catechu L) in Male Rat. MEDISAINS 18 (2), 52-57. doi:10.30595/ medisains.v18i2.7588 spermicidal, antispermatogenic, antiovulatory, anti-implantation, antiestrogenic, and abortifacient activity. The Indian system of medicine, Ayurveda, offers highly promising opportunities when analytical, biological, technological, and clinical advances are collectively integrated with therapeutic rationale based on Ayurvedic principles. A plethora of available data, information, and knowledge on these ingredients could be the subject of newer research interests.

These medicinal ingredients need further reexamination and critical evaluation to explore their lesser known or unknown pharmacological and biological activity/activities and effects. Present-day biotechnological methods could be usefully utilized to evaluate their contraceptive efficacies. There is a need to revive and stimulate new research programs and projects that will not only benefit the need of contraception but will also throw new light on reproductive biology.

\section{AUTHOR CONTRIBUTIONS}

The corresponding author Dr. NB contributed to the concept, initial compilation, structure of the review, and final editing of the text and figures. Co-author Dr. MD contributed to compiling and comparing pharmacological data and the preparation of tables and figures.

\section{ACKNOWLEDGMENTS}

We sincerely thank Dr. Vandana Kozarekar for reference review and edit support.

Azeez, O. I., and Philip, A. A. (2013). Retarded Hippocampal Development Following Prenatal Exposure to Ethanolic Leaves Extract of Datura Metel in Wistar Rats. Niger. Med. J. 54 (6), 411-414. doi:10.4103/0300-1652.126299

Azmeera, M., Elumalai, A., Eswaraiah, M. C., and Mathangi, N. (2012). An Updated Review on Anti-fertility Plants. Inter. J. Pharmacother. 2 (1), 4-6.

Badwaik, H., Giri, T. K., Tripathi, D. K., Singh, M., and Khan, A. H. (2011). A Review on Pharmacological Profile for Phytomedicine Known as Gloriosa Superb Linn. Res. J. Pharmacognosy Phytochemistry 3 (3), 103-107.

Bajaj, A., Mathur, R. S., Wadhwa, M., and Bahel, S. (1981). Effect of Steroidal Fraction of Abrusprecatorius on Testes of Albino Rats. Geobios 8, 29-31.

Barilli, S., Santos, S., and Montanari, T. (2005). Effect of decocyte of northern buchinha fruits (Luffa operculata Cogn.) on female reproduction and embryonic and fetal development. XVII Scientific Initiation Hall. Book of Abstracts (Porto Alegre: Pro-Rectory of Research, UFRGS), 539.

Bhagat, M., and Purohit, A. (1986). Kinetics of the Testicular Cell Population Following Various Curcuma Longa Rhizome Extract Administration in Male Albino Rats, A Morphometric Approach, in: National Entellus. India: National Symposium on the Use of Primates in Biochemical Research Jaipur, 53.

Bhargava, S. K. (1984). Effects of Plumbagin on Reproductive Function of Male Dog. Indian J. Exp. Biol. 22, 153-156.

Bhaskar, V. H., V, H., Profulla, B. R., Kumar, M., and Sangameswaran, B. (2009). Evaluation of the Antifertility Activity of Stem Bark of CrataevanurvalabuchHum. Afr. J. Biotechnol. 8 (22), 6453-6456. doi:10.5897/ajb09.303

Bhavamisra, B. P. (1961). in Varanasi. Chowkhambha Sanskrit Series, IInd Part Chikitsa. 3rd, 7033-7034.

Bidwai, P. P., Wangoo, D., and Bhullar, N. (1990). Antispermatogenic Action of Celastruspaniculatusseed Extract in the Rat with Reversible Change in the Liver. J. Ethnopharmacol 28 (3), 293-303. doi:10.1016/0378-8741(90)90080-d 
Bodhankar, S. L., Garg, S. K., and Mathur, V. S. (1974). Antifertility Screening of Plants. Part IX. Effect of Five Indigenous Plants on Early Pregnancy in Female Albino Rats. Indian J. Med. Res. 62, 831-837.

Brinker, F. (1997). Inhibition of Endocrine Function by Botanical Agents, Antigonadotropic Activity. Br. J. Phytother 4, 123-145.

Bullock, J., Boyle, J., and Wang, M. B. (1995). in Physiology. Editor J. Velker 3rd edn. (Lippincott Williams \& Wilkins), 497-519.

Census of India (2011). Population Projections for India and States 2011 - 2036. Available at: https://nhm.gov.in/New_Updates_2018/Report_Population_ Projection_2019 (Accessed November 6, 2020).

Changamma, C., and Lakshman, J. (2013). Antispermatogenic Effect of Carica Papaya Seed Extract on Steroidogenesis in Albino Rats. Int. J. Pharm. Pharm. Sci. 5 (1), 67-69.

Chaturvedi, M., and Dixit, V. P. (1997). Antifertility Effect of Citrullus colocynthisSchrad in Male Albino Rats. Indian J. Environ. Sci. 1 (2), 89-92.

Chaudhury, M. R., Chandrasekaran, R., and Mishra, S. (2001). Embryotoxicity and Teratogenicity Studies of an Ayurvedic Contraceptive--Pippaliyadivati. J. Ethnopharmacol. Feb 74 (2), 189-193. doi:10.1016/s0378-8741(00)00354-8

Choudhury, P. K., and Jadhav, S. (2013). Pharmacological Action of Plant Alkaloids in Female Reproductive System of Test Animals And/or Human Beings: A Review. Int. J. Pharm. Sci. Rev. Res. 23 (2), 98-107.

Cuomo, Amy. (2010). "Birth Control," in Encyclopedia of Motherhood. Editor A. O'Reilly (Thousand, Oaks, Calif.: Sage Publications), 121-126.

Dahanukar, S. A., Kulkarni, R. A., and Rege, N. N. (2000). Pharmacology of Medicinal Plants and Natural Products. Indian J. Pharmacol. 32, S81-S118.

Das, R. P. (1980). Effect of Papaya Seeds on the Genital Organs and Fertility of Male Rats. Indian J. Exp. Biol. 18, 408-409.

Dash, B., and Basu, R. (1968). Methods for sterilization and Contraception in Ancient and Midieval period. IJHS 3910, 9-24.

De Freitas, T. G., Augusto, P., and Montanari, T. (2005). Effect of Ruta Graveolens L. On Pregnant Mice. Contraception 71 (1), 74-77. doi:10.1016/j.contraception. 2004.07.014

Devendra, N. K., Vijaykumar, B., Malashetty, Y., Seetharam, N., Suresh, P., and Patil, S. B. (2009). Effect of Ethanol Extract of Whole Plant of Trichosanthescucumerina Var. Cucumerina L. On Gonadotropins, Ovarian Follicular Kinetics and Oestrous Cycle for Screening of Antifertility Activity in Albino Rats. Int. J. Morphol. 27 (1), 173-182. doi:10.4067/S0717-95022009000100030

Dhawan, B. N., Dubey, M. P., Mehrotra, B. N., Rastogi, R. P., and Tandon, J. S. (1980). Screening of Indian Plants for Biological Activity: Part-IX. Indian J. Exp. Bio 18, 594-602.

Dheeraj, A. (2011). Anti-fertility Activity of Acacia Leucophploea. Scholars Res. Libr. 3 (3), 411-413.

Dikshith, T. S. S., Raizada, R. B., and Mulchandani, N. B. (1990). Toxicity of Pure Alkaloid of Tylophoraasthmaticain Male Rats. Indian J. Exp. Biol. 28 (3), 208-212.

Dimitriadis, E., Robb, D. L., Liu, Y. X., Enders, A. C., et al. (2003). IL-11 and IL11Rá Immunolocalisation at Primate Implantation Sites Supports a Role for IL11 in Placentation and Fetal Development. Reprod. Biol. Endocrinol. 1 (1), 34. doi:10.1186/1477-7827-1-34

Dixit, V. P., Bhargava, S. K., and Gupta, R. A. (1981). Hyperglycemia Induced Testicular Dysfunction after Chronic Administration of Balanites Roxburghii Planch Fruit Pulp Extract in Dog (Canis Indicus). Indian J. Exp. Biol. 19, 918-921.

Dixit, V. P., and Bhargava, S. K. (1983). Reversible Contraception like Activity of Embelin in Male Dogs (Cannis Indicus Linn.). Andrologia 15 (5), 486-494. doi:10.1111/j.1439-0272.1983.tb00174.x

Dixit, V. P., and Joshi, S. (1983). Effect of Aloe Barbadensis and Clofibrate in Triton Induced Hyperlipidaemic presbytis Monkeys. Ind. J. Med. Res. 78, 417-421.

Dixit, V. P., and Joshi, S. (1982). Effects of Chronic Administration of Garlic (Allium Sativum Linn) on Testicular Function. Indian J. Exp. Biol. 20, 534-536.

Dixit, V. P., Joshi, S., and Kumar, A. (1983). Possible Antispermatogenic Activity of Gloriosa Superba (EtOH-Extract) in Male Gerbil (Merioneshurriane Jerdon): A Preliminary Study. Comp. Physiolecol 8, 17-22.

Dixit, V. P., Sinha, R., and Gupta, I. (1987). Inhibition of Sperm Production and Sperm Dynamics in Abrus precatorius Treated Males. The Indian Zoologist 11 (1-2), 115-118.

Dufau, M. L., Winters, C. A., Hattori, M., Aquilano, D., Baranao, J. L., Nozu, K., et al. (1984). Hormonal Regulation of Androgen Production by the Leydig Cell. J. Steroid Biochem.20, 161-173. doi:10.1016/0022-4731(84)90203-6
Dutta, D. C. (2013). Text Book of Gynaecology Including Contraception. 6th edition. New Delhi: Jaypee Brothers medical publisher.

Elizabeth, S., Ann, B., Jacqueline, E. D., Taylor, R., Lori, S. A., Naomi, L-D., et al. (2020). Report- Adding it up: Investing in Sexual and Reproductive Health 2019. Available at: https://www.guttmacher.org/report/adding-it-up-investingin-sexual-reproductive-health-2019-executive-summary/resources (Accessed November 10, 2020).

Farooq, T., Vanitha Kumari, G., Bhuvaneswari, G., and Malini, T. (1997). Effects of Anethole on Accessory Sex Tissue of Albino Rats. J. Res. Ayurv Siddha 15, 161-170.

Galib, A. C., Kar, M. M., Raoand Ala, N. (2008). Concepts of Contraception in Ancient India \& Status in Present Scenario. J. Ind. Inst. Hist. Med. XXXVIII, 79-88.

Gangadhar, R., and Lalithakumari, K. (1995). Abortifacient Activity of the Aqueous Extract of the Leaves of Aegle Marmelos (Bel) in Albino Rats. Indian Drugs 32, 129-131.

Ganguly, M., Borthakur, M. K., Devi, N., and Mahanta, R. (2007). Antifertility Activity of T Methanolic Leaf Extract of Cissampelos Pareira in Female Albino Mice. J. Ethnopharmacology 111 (3), 688. doi:10.1016/j.jep.2007.01.023

Garg, S. K., Mathur, V. S., and Chaudhary, R. R. (1978). Screening of Indian Plants for Antifertility Activity. Indian J. Exp. Biol. 16, 1077. doi:10.1007/ bf00930383

Garg, S. K. (1975). Antifertility Effect of Some Chromatographic Fractions of Daucas Carota. Indian J. Pharmacol. 7, 40-42.

Garg, S. K. (1974). Antifertility Effect of Oil from Few Indigenous Plants on Female Albino Rats. Planta Med. 26, 391. doi:10.1055/s-0028-1099405

Garg, S. K., and Garg, G. P. (1970). A Preliminary Report on the Smooth Muscle Stimulating Property of Some Indigenous Plants on Isolated Rat Uterus. Bull. P. G. Chandigarh 4, 162.

Garg, S. K., and Garg, G. P. (1971). Antifertility Effects of Areca Catechu Linn. And Carica Papaya Linn. in Female Albino Rats. Indian J. Pharmac 3, 23.

Gark, S. K., Mathur, V. S., and Chaudhury, R. R. (1978). Screening of Indian Plants for Anti-fertility Activity. Indian J. Exp. Biol. 16 (10), 1077-1079.

Gbotolorun, S. C., Osinubi, A. A., Noronha, C. C., and Okanlawon, A. O. (2008). Antifertility Potential of Neem Flower Extract on Adult Female SpragueDawley Rats. Afr. Health Sci. Sep. 8 (3), 168-173.

Gediya, S., Ribadiya, C., Soni, J., Shah, N., and Jain, H. (2011). Herbal Plants Used as Contraceptives. Int. J. Curr. Pharm. Rev. Res. 2 (1), 47-53.

Georgia Institute of Technology (2019). Contraceptive Jewelry Could Offer a New Family Planning Approach Science Daily. Available at: www.sciencedaily.com/ releases/2019/03/190326105705.htm (Accessed November 14, 2020).

Ghosh, A. K., Das, A. K., and Patra, K. K. (2011). Studies on Antifertility Effect of Rhizome of Curcuma Longa linn. Asian J. Pharm. Life Sci. 1 (4), 349-353.

Goel, R. K., Prabha, T., Kumar, M. M., Dorababu, M., Prakashand Singh, G. (2006). Teratogenicity of Asperagusracemosus Wild. Root, an Herbal Medicine. Indian J. Exp. Biol. 44 (7), 570-573.

Goncim, H. Y., Mador, E. S., and Ogunranti, J. O. (2010). Ricinus Communis Var Minor Inhibits Follicular Development and Possibly Ovulation in Human Subjects as Shown by Ultrasound Follicle Tracking. Clinical Medicine Insights. January: Reproductive Health.

Gupta, A. K., Bindal, M. C., Gupta, S. K., Prakash, D., and Vedpal (2013). Aphrodisiac Activity of Semecarpus Anacardium Nut. Int. Res. J. Pharm. 4, 202-204.

Gupta, I., Tank, R., and Dixit, V. P. (1985). Fertility Regulation in Males: Effect of Hibiscus Rosa-Sinensis and Malvaviscus Flower Extract on Male Albino Rats. Proc. Nat. Acad. Sci. India 55 (B), 262-267.

Gupta, M., Mazumder, U. K., Pal, D. K., and Bhattacharya, S. (2003). Antisteroidogenic Activity of Methanolic Extract of Cuscutareflexa Roxb. Stem and Corchorus Olitorius Linn. Seed in Mouse Ovary. Indian J. Exp. Biol. 41, 641-644.

Gupta, R. S., Choudhary, R., Yadav, R. K., Verma, S. K., and Dobhal, M. P. (2005a). Effect of Saponins of Albizia Lebbeck (Linn.) Benth. Bark on the Reproductive System of Male Albino Rats. J. Ethnopharmacol 96 (1-2), 31-36. doi:10.1016/j. jep.2004.07.025

Gupta, R. S., and Dixit, V. P. (1989). Testicular Cell Population Dynamics Following Palmitine Hydroxide Treatment in Male Dogs. J. Ethnopharmacol 25, 151-157. doi:10.1016/0378-8741(89)90016-0

Gupta, R. S., Dobhal, M. P., and Dixit, V. P. (1996). Morphometric and Biochemical Changes in Testes of Presbytis Entellus Dufresne (Langur 
Monkey) Following Aristolochic Acid Administration. Ann. Biol. 12 (2), 328-334.

Gupta, R. S., Kachhawa, J. B., and Chaudhary, R. (2004). Antifertility Effects of Methanolic Pod Extract of Albizia Lebbeck (L) Benth in Male Rats. Asian J. Androl. 6 (2), 155-159.

Gupta, R. S., Kumar, P., and Dixit\&Dhobhal, V. P. M. P. (2000). Antifertility Studies of Root Extract of BarleriaprionitisLinn. In Male Albino Rats with Special Reference to Testicular Cell Population Dynamics. JEthnopharmacol 70 (2), 111-117. doi:10.1016/s0378-8741(99)00150-6

Gupta, R. S., and Sharma, A. (2003). Antifertility Effect of Tinospora Cordifolia Willd. Stem Extract in Male Rats. Indian J. Exp. Biol. 41, 885-889.

Gupta, R. S., Sharma, N., and Dixit, V. P. (1990). Calotropin, A Novel Compound for Fertility Control. Ancient Sci. Life 9 (4), 224-230.

Gupta, R. S., Sharma, R., Sharma, A., Choudhary, R., Bhatnagar, A. K., and Joshi, Y. C. (2005b). Antifertility Effects of Pueraria Tuberosa Root Extract in Male Rats. Pharm. Biol. 42 (8), 603-609. doi:10.1080/13880200490902491

Gupta, R. S., Kanwar, M., Rehwani, H., Verma, S. K., and Dobhaal, M. P. (2006). Contraceptive Efficacy of Strychnospotatorum Seed Extract in Male Albino Rats. Asian J. Exp. Sci. 20 (1), 181-187.

Gupta, S., Dheeraj, A., Sharma, N. K., Jhade, D., and Bharti, A. (2011). Effect of Plumbagin Free Alcohol Extract of Plumbago Zeylanica Linn. Root on Reproductive System of Female Wister Rats. Asian Pac. J. Trop. Med. 4 (12), 978-984. doi:10.1016/S1995-7645(11)60230-7

Gupta, S. S. (1994). Prospects and Perspectives of Natural Plants Products in Medicine. Indian J. Pharmacol. 26, 1-12.

Gupta, S., Sanyal, S. N., and Kanwar, U. (1989). Antispermatogenic Effect of Embelin, a Plant Benzoquinone, on Male Albino Rats In Vivo and In Vitro. Contraception 39, 307-320. doi:10.1016/0010-7824(89)90063-2

Gutiérrez-Pajares, J. L., Zúñiga, L., and Pino, J. (2003). Ruta Graveolens Aqueous Extract Retards Mouse Preimplantation Embryo Development. Reprod. Toxicol. 17 (6), 667-672. doi:10.1016/j.reprotox.2003.07.002

Hadimur, K., Revansiddppa, S. S., Lone, N. D., Veena, K., and Neelamma, P. (2014). Anti-implantation and Pregnancy Interruption Activity of Japakusuma (Hibiscus Rosa Sinensis) \& its Combinations in Albino Rats. Br. J. Med. Health Res. 1 (3), 11-18. doi:10.7897/2277-4343.04316

Indradev, T. (1998). Rasaratnasamuccaya of Rasavagbhata. 1st Edition. Varanasi, India: Chaukhambha Sanskrit Bhavan, 95-96.

Isichei, C. O., Das, S. C., Ogunkeye, O. O., Okwuasaba, F. K., Uguru, V. E., Onoruvwe, O., et al. (2000). Preliminary Clinical Investigation of the Contraceptive Efficacy and Chemical Pathological Effects of RICOM-1013-J of Ricinus communis Var Minor on Women Volunteers. Phytother Res. 14 (1), 40-42. doi:10.1002/(sici)1099-1573(200002)14:1

Jain, G. C., and Dixit, V. P. (1982). Effect of Annona Squamosa Ethanol Extract and Testicular Function of Dogs (Canis Indicus Linn.), II Annual Session of Science, 22.

Jamwal, K. S., and Anand, K. K. (1962). Preliminary Screening of Some Reputed Abortifacient Indigenous Plants. Indian J. Pharm. 2, 218-220.

Jansen, G. C., and Wolhlmuth, H. (2014). Carrot Seed for Contraception: A Review. Aust. J. Herbal Med. 26, 10-17.

Jati, K. P., Mahapatra, A. K., and Rajagopala, S. (2018). Teratogenic Effect of Herbal Drugs -A Review. IJAAR Volume III Issue X Sep -Oct, 1516-1523.

Jha, R. K., and Dixit, V. P. (1986). Inhibition of Spermatogenesis after Chronic Administration of Terminelia Arjuna and Sapindustrifoliatus(50\% EtOH Extract) in Male Albino Rats. Proc. Nat. Acad. Sci. 56 (3), 94-99.

Jon, K. (2012). A History of Birth Control Methods. Planned Parenthood Report. January, Federation of America. Available at: https://www.plannedparenthood. org/files/2613/9611/6275/History_of_BC_Methods.pdf (Accessed November 20, 2020).

Jugnu, S., and Sharma, B. (2011). Rajamartanda. Ancient Sanskrit Medical Text of Maharaja Bhoja with Sanskrit Text: English Transliteration and Commentary in English. Varanasi: Chaukhambha Orientalia. StreeRogadhikara, 16 to 18 and 31 .

Kalita, J. C., Chakrabarty, A., and Tanti, B. (2011). Assessment of Antifertility Activity of Some Traditionally Used Plants by Different Ethnic Communities in Three Districts of Assam, India. J. Herbal Med. Toxicol. 5 (2), 65-72.

Kamboj, V. P., and Dhawan, B. N. (1982). Research on Plants for Fertility Regulation in India. J. Ethnopharmacology 6 (2), 191-226. doi:10.1016/03788741(82)90004-6
Kasturi, M., Nazeer, A. R., Pathan, K. M., Parveen, D. S., and Manivannan, B. (1997). Effects of Azadirachtaindica Leaves on the Seminal Vesicles and Ventral Prostate in Albino Rats. Indian J. Physiolpharmacol 41, 234-240.

Kaur, R., Sharma, A., Kumar, R., and Kharb, R. (2011). Rising Trends towards Herbal Contraceptives. J. Nat. Product. Plant Resour. 1 (4), 5-12.

Keshri, G., Singh, M. M., Lakshmi, V., and Kamboj, V. P. (1995). Post-coital Contraceptive Efficacy of the Seeds of Nigella Sativa in Rats. Indian J. Physiolpharmacol 39, 59-62.

Kholkute, S. D., Kekere, M. B., and Munshi, S. R. (1979). Antifertility Effect of Fruits of Piper Longum on Female Rats. Indian J. Exp. Biol. 17, 289-290.

Khouri, N. A., and Akawi, Z. E. (2005). Antiandrogenic Activity of Ruta Graveolens L in Male Albino Rats with Emphasis on Sexual and Aggressive Behaviour. Neuroendocrinology Lett. 26 (6), 823.

Kuchimara (2007). Kuchimaratantra, Text with English Translation by Goli Penchalaprasada. Varanasi: Chaoukhambha Krisandas Academy.

Kulshreshtha, S. S., and Mathur, R. S. (1990). Effect of Steroidal Fraction of Seeds of AbrusprecatoriusLinn. On Rat Testis. Indian J. Exp. Biol. 28, 752-756.

Kumar, C. P., and Sachin, J. (2013). Pharmacological Action of Plant Alkaloids in Female Reproductive System of Test Animals And/or Human Beings: A Review. Int. J. Pharm. Sci. Rev. Res. 23, 98-107.

Lakmipatishashtri (1983). "Varanasi. Yogaratnakara - Yonivyapat," in Chikitsa Yonivyapad/Garbhsnivaran 1, 4-6. 3rd ed. (Chowkhambha Sanskrit Series).

Lal, B., and Udupa, K. N. (1993). A Preliminary Study of Antifertility Effect of an Indigenous Drug -Arjuna (Terminalia Arjuna). JRAS 14 (1), 165-169.

Lal, B., Udupa, K. N., and Tripathi, V. K. (1992). Study of the Antifertility Effect of Nirgundi [Vitex Nirgundo\}- A Preliminary Trails. J. Res. Ayurved Siddha 13 (12), 89-93.

Latha, K. P., Kirana, H., and Girish, H. N. (2013). Anti Implantation Activity of the Hydrolcoholoc Tuber Extract of Gloriosa Superb Linn in Female Albino Rats. Int. J. Adv. Pharm. Biol. Chem. 2 (3), 443-448.

Laxmi, V., Kumar, R., Agarwal, S. K., and Dhar, J. D. (2006). Antifertility Activity of Piper Longum in Female Rats. NAT. Prores 20 (3), 235-239. doi:10.1080/ 14786410500045465

Lipsey, R. G., Carlaw, K., and Bekar, C. (2005). Historical Record on the Control of Family Size. Economic Transformations: General Purpose Technologies and Long-Term Economic Growth. Oxford University Press, 335-340.

Lobat, J., Neda, S., Najmeh, S., Mehrnoosh, S., Soleiman, K., Hedayatollah, S., et al. (2015). Antioxidant Activity and Teratogenicity Evaluation of LawsoniaInermis in BALB/c Mice. J. Clin. Diagn. Res. 9 (5), FF01-FF04. doi:10.7860/JCDR/2015/ 12290.5911

Londonkar, R. L., Srinivasreddy, P., Somanathreddy, P., and Patil, S. B. (1998). Nicotine Induced Inhibition of Activities of Accessory Reproductive Ducts in Male Rats. J. Ethnopharmacol 60 (30), 215-221. doi:10.1016/s0378-8741(97) 00148-7

Makonnen, E., Zerihun, L., Assefa, G., and Rostom, A. A. (1999). Antifertility Activity of Ricinus communis Seed in Female guinea Pigs. East. Afr. Med. J. 76, 335-337.

Malhi, B. S., and Trivedi, V. P. (1972). Vegetable Antifertility Drugs of India. Q. J. Crude Drug Res. 12 (3), 1922-1928. doi:10.3109/13880207209068244

Mali, P. C. (1999). Antifertility Activity of Euphorbia neriifoliaLinn. Root Extract in Male Rats. Indian J. Environ. Sci. 3 (2), 85-190.

Malini, T., Manimaran, R. R., Arunkumar, J., Aruldhas, M. M., and Govindarajulu, P. (1999). Effects of Piperine on Testis of Albino Rats. J. Ethnopharmacology 64 (3), 219-225. doi:10.1016/s0378-8741(98)00128-7

Malpani, A., and Mahurkar, N. (2018). Antifertility Activity of Different Extracts of Tuberous Roots of Gloriosa Superba Linn. In female Wistar albino rats Indian Drugs 55 (7), 67-71.

Mandal, R., and Dhaliwal, P. K. (2007). Antifertility Effect of Melia Azedarach Linn. (Dharek) Seed Extract in Female Albino Rats. Indian J. Exp. Biol. 45, 853-860.

Mandal, T. K., and Das, N. S. (2010). Testicular Toxicity in Cannabis Extract Treated Mice: Association with Oxidative Stress and Role of Antioxidant Enzyme Systems. Toxicol. Ind. Health 26, 11-23. doi:10.1177/0748233709354553

Maurya, R., Srivastava, S., Kulshreshta, D. K., and Gupta, C. M. (2004). Traditional Remedies for Fertility Regulation. Curr. Med. Chem. 11 (11), 1431-1450. doi:10. 2174/0929867043365215 
Mint (2020). The Unmet Need for Contraception in India. Available at: https:// www.livemint.com/news/india/the-unmet-need-for-contraception-in-india11581350642826.html (Assessed November 5, 2020).

Mishra, R. K., and Singh, S. K. (2009). Antispermatogenic and Antifertility Effects of Fruits of Piper Nigrum L. In Mice. Indian J. Exp. Biol. 47, 706-714.

Misra, D. N., and Shukla, G. D. (1980). Vitafix in Premature Ejaculation A Controlled Trial. Indian Pract. 33, 81.

Mukherjee, P. (2002). An Approach to Evaluation of Botanicals. 1st edn. New Delhi, India: Business Horizones; Quality Control Herbal Drugs.

Munshi, S. R., Shetye, T. A., and Nair, R. K. (1977). Antifertility Activity of Three Indigenous Plant Preparations. Plant Med. 31, 73-75. doi:10.1055/s-00281097494

Mutreja, A., Agarwal, M., Kushwaha, S., and Chauhan, A. (2008). Effect of Nelumbo nucifera Seeds on the Reproductive Organs of Female Rats. Iranian J. Reprod. Med. 6 (1), 7-11.

Nand, O. P. (1981). Antifertility Investigation on Embelin- an Oral Contraceptive of Plant Origin. Plant Med. 41 (3), 259-266. doi:10.1055/s-2007-971712

Naseem, M. Z., Patil, S. R., Patil, S. R., and Patil, S. B. (1998). Antispermatogenic and Androgenic Activities of Momordica Charantia(Karela) in Albino Rats. JEthnopharmacol 61 (1), 9-16. doi:10.1016/s0378-8741(98)00006-3

Neeru, V., and Sharma, S. K. (2008). Post-coital Antifertility Activity of Hibiscus Rosa-Sinensis Linn. Roots. Evid. Based Complement. Alternat Med. 5 (1), 91-94. doi:10.1093/ecam/nem003

Novaro, V., Gonzalez, E., Jawerbaum, A., Rettori, V., Canteros, G., and Gimeno, M. F. (1997). Nitric Oxide Synthase Regulation during Embryonic Implantation. Reprod. Fertil. Develop. 9 (5), 557-564. doi:10.1071/r97005

Ola-Mudathir, K. F., Suru, S. M., Fafunso, M. A., Obioha, U. E., and Faremi, T. Y. (2008). Protective roles of onion and garlic extracts on cadmiuminduced changes in sperm characteristics and testicular oxidative damage in rats. Food Chem. Toxicol. 46 (12), 3604-3611. doi:10.1016/j. fct.2008.09.004

Pal, Ria., Arup, M., and Achintya, S. (2013). Exploring post Coital Antifertility Activity with Toxicological and Hormonal Profiling of Sapindustrifoliatus Linn. Int. Res. J. Pharm. Appl. Sci. 3 (5), 53-60.

Pal, A. K., Bhattacharya, K., Kabir, S. N., and Pakrashi, A. (1985). Flowers of Hibiscus rosa-sinensis, a potential source of contragestative agent: II. Possible mode of action with reference to anti-implantation effect of the benzene extract. Contraception 32 (5), 517-529. doi:10.1016/0010-7824(85) 90021-6

Palep, H. S., and Jukar, S. R. (2003). Effectiveness of Indigenous Oral Contraceptive. Available at: https://www.bhj.org.in/journal/2003_4502_april/ effectiveness_310.htm (Accessed October 15, 2020).

Pande, D., Malik, S., Bora, M., and Srivastav, P. (2002). A rapid protocol for in vitro micropropagation of Lepidium sativum linn. and enhancement in the yield of lepidine. In Vitro Cell Dev Biol -Plant 38, 451-455. doi:10.1079/IVP2002322

Pandey, G., and Madhuri, S. (2010). Pharmacological Activities of Ocimum Sanctum (Tulsi): A Review. Int. J. Pharm. Sci. Rev. Res. 5 (1), 61.

Pathak, A. K., Mallurwar, V. R., Kondalkar, A. K., and Soni, S. (2005). A Review of Plants with Anti-fertility Activity. Nig J. Nat. Prod. Med. 09, 4-10. doi:10.4314/ njnpm.v9i1.11824

Pathak, S., Jonathan, S., and Prakash, A. O. (1995). Timely Administration of Extract of Ferulajaeschkena Causes Luteolysis in the Ovary of Cyclic Guineapig. Indian J. Physiolpharmacol 39, 395-399.

Patil, S. J., and Patil, S. B. (2013). Antiovulatory Activity of Petroleum Ether Extract of Chromatographic Fractions of Citrus Medica Seeds in Albino Rats. Int. J. Med. Sci. 13 (6), 410-417. doi:10.3923/jms.2013.410.417

Pillai, N. R., Alam, M., and Purushothaman, K. K. (1982). Studies on Antifertility Activity of Oleanolic Acid 3a-Glucoside (RDG-D). J. Res. Indian Med. Yoga Homeop 12, 26-29.

Pillai, N. R., Alam, M., and Purushothaman, K. K. (1977). Studies on the Antifertility Activity of Oleanolic Acid 3-\$-glucoside (RDG-1). J. Res. Indian Med. Yoga Homeopath. 12, 26-29. doi:10.1016/0360-1323(77)90003-8

Pokharkar, R. D., Saraswat, R. K., and Kotkar, S. (2010). Survey of Plants Having Antifertility Activity from Western Ghat Area of Maharashtra State. J. Herbal Med. Toxicol. 4 (2), 71-75.

Prakash, A. O., Kushwah, K., and Pathak, S. (1989). Effect of Ethanolic Extract of Ferula Jaeschkeana Vatke on the Biochemical Constituents in Vital Organs of Pregnant Rats. Indian J. Pharmacol. 21, 129-134.
Prakash, A. O., Saxena, V., Shukla, S., and Mathur, R. (1985). Contraceptive Potency of Pueraria Tuberosa D.C. And its Hormonal Status. Acta Eur. Fertil. 16 (1), 59-65.

Prakash, S. S. (1981). Anti-fertility Investigations on Embellin. Planta Med. 41, 259-266. doi:10.1055/s-2007-971712

Priya, G., Saravanan, K., and Renuka, C. (2012). Medicinal Plants with Potential Antifertility Activity- A Review of Sixteen Years of Herbal Medicine Research (1994-2010). Int. J. PharmTech Res. 4 (1), 481-494.

Purohit, A., Vyas, S. K., and Vyas, K. B. (2008). Contraceptive Efficacy of Plumbago Zeylanica Root Extract (50\% Etoh) in Male Albino Rats with Special Emphasis on Testicular Cell Population Dynamics. Anc Sci. Life Jan 27 (3), 31-35.

Raj, A., Singh, A., Sharma, A., Singh, N., Kumar, P., and Bhatia, V. (2011). Antifertility Activity of Medicinal Plants on Reproductive System of Female Rat. Int. J. BioEngineering Sci. Technol. 02 (03), 44-50.

Rajeshwaradatta, S. (2001). Bhaishjya Ratnavali of Govindadass with 'Vidyotinl' Hindi Commentary. 14th Edition. Varanasi: Chaukhambha Sanskrit Sansthan. VerseYonivyiipad 67/27, 28, 30-32.

Reda, H., El, M., and Azza, A. (2018). Attia Ginger Causes Subfertility and Abortifacient in Mice by Targeting Both Estrous Cycle and Blastocyst Implantation without Teratogenesis. Phytomedicine 50, 300-308. doi:10. 1016/j.phymed.2018.01.021

Reddy, M. C., Murthy, R. K. D., and Saraswati, B. P. (1997). Antispermatogenic and Androgenic Activities of Various Extracts of Hibiscus Rosa-Sinensis in Albino Mice. Indian J. Exp. Biol. 35, 1170-1174.

Roop, J. K., Dhaliwal, P. K., and Guraya, S. S. (2005). Extracts of Azadirachta indica and Melia Azadarach Seeds Inhibit Folliculogenesis in Albino Rats Braz. J.Med.Bio.Res 38 (6), 943-947. doi:10.1590/s0100$879 \times 2005000600017$

Roy, C. A., and Venkatakrishna, B. H. (1983). Impairment of Spermatogenesis by Cichorium Intybus Plant Extract. Naturwiss enschaften 70, 365-369.

Saksena, S. K. (1971). Study of Antifertility Activity of the Leaves of Momordica linn (Karela). Indian J. Physiol. Pharmacol. 15 (2), 79-80.

Salunke, K. R., Ahmed, R. N., and Marigoudar, S. R. L. (2011). Effect of Graded Doses of Caesalpinia Bonducella Seed Extract on Ovary and Uterus in Albino Rats. J. Basic Clin. Physiol. Pharmacol. 22 (1-2), 49-53. doi:10.1515/jbcpp. 2011.006

Samajdar, S., and Ghosh, A. K. (2017). Pharmacological effects of Sesbania sesban Linn : An overview. PharmaTutor 5 (7), 16-21.

Samatha, J., and Bhattacharya, S. (2011). Cissampelos Pareira: A Promising Antifertility Agents. Int. J. Res. Ayurveda Pharm. 2 (2), 439-442.

Sandhyakumary, K., Bobby, R. G., and Indira, M. (2003). Antifertility Effects of Ricinus communis Linn. On Rats. Phytother Res. 17 (5), 508-511. doi:10.1002/ ptr. 1308

Sarkar, M., Gangopadhyay, P., Basak, B., Chakrabarty, K., Banerji, J., Adhikary, P., et al. (2000). The Reversible Antifertility Effect of Piper BetleLinn. On Swiss Albino Male Mice. Contraception 62, 271-274. doi:10.1016/s0010-7824(00) 00177-3

Sarwat, J., Salma, R., Mir, A. K., Mushtaq, A., Muhammad, Z., Muhammad, A., et al. (2009). Antifertility Effects of Ethanolic Seed Extract of Abrusprecatorius L. On Sperm Production and DNA Integrity in Adult Male Mice. J. Med. Plants Res. 3 (10), 809-814.

Sathiyaraj, K., Sivaraj, A., Madhumitha, G., Vinoth kumar, P., Mary saral, A. M., Devi, K., et al. (2010). Antifertility Effect of Aqueous Leaf Extract of Aegle Marmelos on Male Albino Rats. Int. J. Curr. Pharm. Res., 2 (1), 26-29.

Satvalekar, S. D. (1958b). Atharva Veda Ka Svadhyaya, Part II, (Kand VI - 138 / 1, 4, 5). 2nd. Pardi: Swadhyaya Mandal.

Satvalekar, S. D. (1958a). Atharva Veda Ka Svadhyaya, Part II, (Kand VI - 37 / 2,3). 2nd edition. Pardi: Swadhyaya Mandal.

Satyawati, G. V. (1983). Indian Plants and Plant Products with Antifertility Effect [A Review of Edition Literature between 1975-1982. New Delhi: ICMR.

Schulz, V., Hänsel, R., and Tyler, V. (2001). Rational phytotherapy: a physician's guide to herbal medicine. Psychology Press.

Seshadri, C., and Pillai, S. R. (1981). Antifertility Activity of a Compound Ayurvedic Preparation. J. Sci. Res. Pl Med. 2 (1\&2), 1-3.

Shah, N. V., and Varute, A. J. (1980). Effect of Daucus Carrota Seed Extract on Male Reproductive Organs of Albino Rats (testisII). in: All India Symposium in Life Sciences. Nagpur 91, 217. 
Shah, S. K., Jhade, D., and Chouksey, R. (2017). Pharmacological Evaluation and Antifertility Activity of Aloe Barbadensis Linn on Female Wistar Rats. Int. J. Phytomedicine 9 (2), 253-260.

Shah, S. K., Jhade, D., and Chouksey, R. (2016). Antifertility Activity of Ethanolic and Aqueous Extracts of Aloe Vera Mill on Female Wistar Rats: Rising Approaches of Herbal Contraception. J. Pharm. Sci. Res. 8 (9), 952-957.

Sharma, A., Verma, P. K., and Dixit, V. P. (2003). Effect of Semecarpus Anacardium Fruits on Reproductive Function of Male Albino Rats. Asian J. Androl. 5, 121-124.

Sharma J, J., Sharma, S., and Jain, R. (2001). "Antifertility Activity of Cuminum Cyminum on Reproductive Organs of Male Albino Rats (Rattus norvegicus)," in National Symposium Reproductive Biology and Comparative Endocrinology Vadodara (Gujarat, 69.

Sharma, J. D., Jha, R. K., Gupta, I., and Jain, P. (1987). Antiandrogenic Properties of Neem Seed Oil Azadirachta indica in Rat and Rabbit. Ancient Sci. Life 1, 30-38.

Sharma, J. D., Sharma., L., and Yadav., P. (2007). Antifertility Efficacy of Piper Betel Linn. (Petiol) on Female Albino Rats. Asian J.Exp.Sci. 21 (1), 145-150.

Sharma, N., and Jacob, D. (2001b). Inhibition of Fertility and Functional Alteration in the Genital Organs of Male Swiss Albino Mouse after Administration of Calotropis Procera Flower Extract. Pharm. Biol. 39 (6), 403-407. doi:10.1076/ phbi.39.6.403.5882

Sharma, N., and Jacob, D. (2001a). Antifertility Investigation and Toxicological Screening of the Petroleum Ether Extract of the Leaves of Mentha Arevensis L. In Male Albino Mice. J. Ethnopharmacology 75 (1), 5-12. doi:10.1016/s03788741(00)00362-7

Sharma Rs, R. S., Rajalakshmi, M., and Jeyaraj, D. A. (2001). Current Status of Fertility Control Methods in India. J. Biosci. 26, 391-305. doi:10.1007/ bf02704741

Sharma, S., Mehta, B. K., and Gupta, D. N. (1994). Screening of post-coital Antiimplantation Activity of Machelachampaka (Anthers) and Centratherumanthelminticum (Seeds). Indian Drugs 31, 280-281.

Sheeja, E., Joshi, S. B., and Jain, D. C. (2011). Anti-ovulatory and Estrogenic Activity of Plumbago Rosea Leaves in Female Albino Rats. Indian J. Pharmacol. 41 (6), 273-277. doi:10.4103/0253-7613.59927

Shibeshi, W., Makonnen, E., Zerihun, L., and Debella (2006). Effect of Achyranthes aspera L. on fetal abortion, uterine and pituitary weights, serum lipids and hormones. Afr. Health Sci. 6 (2), 108-112. doi:10.5555/afhs.2006.6.2.108

Shrestha, J., Shanbhag, T., Shenoy, S., Amuthan, A., Prabhu, K., Sharma, S., et al. (2010). Antiovulatory and Abortifacient Effects of Areca Catechu (Betel Nut) in Female Rats. Indian J. Pharmacol. 42 (5), 306-311. doi:10.4103/0253-7613.70350

Shrivastava, S., Dwivedi, S., Dubey, D., and Kapoor, S. (2007). Traditional Herbal Remedies from Madhya Pradesh Used as Oral Contraceptives- A Field Survey. Int. J. Green Pharm. 1 (1), 18-22.

Singh, A. R., Singh, K. P., and Shekhawat, S. (2013). Spermicidal Activity and Antifertility Activity of Ethanolic Extract of Withaniasomnifera in Male Albino Rats. Int. J. Pharm. Sci. Rev. Res. 21 (2), 227-232.

Singh, S. P. (1990b). Effect of Cinnamomum Camphora Leaf Extract on Testicular Function of House Sparrow (Passer domesticus L). Indian J. Phy Nat. Sci. 10, $22-25$.

Singh, S. P. (1985). Regulation of Fertility in Male through an Indigenous Plant Semecarpus Anacardium Linn. J. Res. Edu Indian Med. 4 (384), 9-20.

Singh, S. P. (1990a). Fertility Control of Female through Sesbania Sesbanseeds. J. Res. Educ. Indian Med. 9 (4), 227-232. doi:10.1071/sr9900227

Sinha, K. C., Rair, S. S., Bardhan, J., Thomas, P., Jain, A. K., and Jain, R. K. (1984). Anti-implantation Effect of Neem Oil. Indian J. Med. Res. 80, 708-710.

Sinha, R. (1990). Post-testicular Antifertility Effects of Abrusprecatoriusseed Extract in Albino Rats. J. Ethnopharmacol 28 (2), 13-81. doi:10.1016/03788741(90)90027-q

Sinha, R. K., and Nathawat, G. S. (1989). Anti-fertility Effects of Some Plants Used by the Street Herbal Vendors for Birth Control. Ancient Sci. Life (2), 66-68.

Soni, P., Siddiqui, A. A., Dwivedi, J., and Soni, V. (2013). Antiovulatory and Estrogenic Activity of Stem of Musa Paradisiaca in Female Albino Rats. J. Appl. Pharm. Sci. 3 (08), 102-106. doi:10.5667/tang.2013.0011

Soni, P. K., Luhadia, G., Sharma, D. K., and Mali, P. C. (2015). Antifertility Activates of Traditional Medicinal Plants in Male with Emphasis on Their Mode Action: a Review. J. Glob. Biosci. 4, 1165-1179.

Srivastava, A., Srivastava, V. K., and Singh, G. (2017). Study of Teratogenic Effects of Chitrak (Plumbago Zeylanica) an Ayurvedic Drug on Developing Mice
Embryo. J. Adv. Res. Ayur. Yoga Unani Sidd. Homeo. 4 (1\&2), 46-50. doi:10. 24321/2394.6547.201711

Sur, T. K., Pandit, S., and Pramani, K. T. (1999). Antispermatogenic Activity of Leaves of Aegle Marmelos Corr. In Albino Rats: A Preliminary Report. Biomedicine 19, 199-202.

Sur, T. K., Pandit, S., Pramanik, T., and Bhattacharyya, D. (2002). Effect of Aegle Marmelos Leaf on Rat Spermmotility: an Invitro Study. Indian J. Pharmacol. 34, 276-277.

Sushma, Y., Kulkarni, G., and Singh, S. (2016). Antifertility Activity of Aqueous and Ethanolic Extracts of Semecarpus Anacardium Fruit in Female Albino Rats. Int. J. Pharm. Sci. Res. 7 (3), 1235-1239.

Sushrut (2002). Susrutsamhita with Nibandhasangraha of Dalhans [Ayurved Classical Text]. 7th Edition. Varanasi, India: Chaukhambha Orientalia.

Tamboli, S. A., and Konadawar, M. S. (2013). Anti-Implantation Activity of the Leaf Extract of Ailanthus ExcelsaRoxb. Int. J. Pharm. Pharm. Sci. 5 (Suppl. 4), $128-129$.

Taprial, S., Kashyap, D., Mehta, V., Kumar, S., and Kumar, D. (2013). Antifertility effect of hydroalcoholic leaves extract of Michelia champaca L.: an ethno medicine used by Bhatra women in Chhattisgarh state of India. $J$. Ethnopharmacol. 147 (3), 671-675. doi:10.1016/j.jep.2013.03.003

Tewari, P. V. (1974). Preliminary Clinical Trial on Flowers of Hibiscus Rosasinensis as an Oral Contraceptive. J. Res. Indian Med. Yoga Homeopath 9, 96-98

Tewari, P. V., Sharma, S. K., and Basu, K. (1976). Clinical Trial of an Indigenous Drug as an Oral Contraceptive. J. Natl. Integrated Med. Assoc. 18, 117-118.

Tewari, P. V., and Chaturvedi, C. (1981). Method of Population Control in Ayurvedic Classics. Ancient Sci. Life, I, 72-79.

Tewari, P. V., Mapa, H. C., and Chaturvedi, C. (1971). J. Res. Indian Med. 6 (2), 112.

Thakare, V. N., Kothavade, P. S., Dhote, V. V., and Deshpande, A. D. (2009). Antifertility Activity of Ethanolic Extract of Allium cepa Linn in Rats. Int J. Pharm Tech Res. 1 (1), 73-78.

Tripathi, I. (1969). Gadanigraha of Sodhal with' Vidyotini' Hindi Commentary. 1 st Edition. Verse- Uttarardha; Pradaradhikar: Varanasi. Chaukhambha Sanskrit series, 60-62.

Turner, D. C. (1971). General Endocrinology. 4th ed. Tokyo: WB Saunders Company, Topan Company Ltd.

Udoh, P., and Ekpenyong, J. (2001). Effect of Mucuna Urens(horse Eyes Bean) on the Gonads of Male guinea Pigs. Phytother Res. 15 (2), 99-102. doi:10.1002/ ptr.699

Vagbhatt (2000). Ashtanghridaya with Sarvagasundara of Arundatta and Hemadri [Ayurved Classical Text]. Varanasi: Krishnadas Academy. Verse-Sharira $1 / 8$.

Vanithakumari, G., Manonayagi, S., Padma, S., and Malini, T. (1989). Antifertility Effect of Bambusaarundinaceae Shoot Extracts in Male Rats. J. Ethnopharmacology 25, 173-180. doi:10.1016/0378-8741(89)90019-6

Vasudeva, N., and Sharma, S. K. (2008). Post-coital Antifertility Activity of Hibiscus RosaSinensis Linn. Roots. Adv. Access Publ. 5 (1), 91-94. doi:10.1093/ecam/nem003

Vasudeva, N., and Sharma, S. K. (2006). Post-Coital Antifertility Activity of Achyranthes aspera linn. Root. J. Ethnopharmacology 107 (2), 179-181. doi:10.1016/j.jep.2006.03.009

Verma, S., and Yadav, A. (2021). Rising trends towards the development of oral herbal male contraceptive: an insight review. J. Pharm. Sci. 7, 23. doi:10.1186/ s43094-020-00154-7

Vyas, V., and Purohit, A. (2018). Contraceptive Effect O F Neem Seed Oil and its Active Fractions on Female Albino Rabbits. Asian J. Pharm. Clin. Res. 11 (12), 421-424. doi:10.22159/ajpcr.2018.v11i12.28188

Wati, B. T., and Verute, A. T. (1988). Butea Monosperma Leaf Extract Induced Alterations in the Testicular Function of Albino Rats: A Histological and Biochemical studyInternational Symposium on Recent Advances in Male Reproduction. Hyderabad 12 (14), 16.

Williamson, E. M., Okpako, D. T., and Evans, F. J. (1996). Pharmacological Methods in Phytotherapy Research: Selection Preparation and Pharmacological Evaluation of Plant Material. London: John Wiley \& Sons, 191-212.

World Health Organization (2020). Contraceptives. Available at: https://www.who. int/news-room/fact-sheets/detail/family-planning-contraception (Accessed November 8, 2020).

World Population Clock (2021). World Population Clock: 7.9 Billion People. Worldometer. Available at: https://www.worldometers.info (Accessed March 28, 2021). 
Yadav, R., and Jain, G. C. (2009). Antifertility Effect of Aqueous Extract of Seeds of Cassia Fistula in Female Rats. Adv. Contraception 15, 293-301. doi:10.1023/a: 1006784224191

Yang, Z. M., Le, S. P., Chan, D. B., and Harper, M. J. (1994). Temporal and Spatial Expression of Leukemia Inhibitory Factor in Rabbit Uterus during Early Pregnancy. Mol. Reprod. Develop. 38 (2), 148-152. doi:10.1002/mrd. 1080380205

Conflict of Interest: Author NB is Owner / Director of the company CRIA Consultants Pvt. Ltd., Mumbai (India).
The remaining author declares that the research was conducted in the absence of any commercial or financial relationships that could be construed as a potential conflict of interest.

Copyright (C) 2021 Bhatt and Deshpande. This is an open-access article distributed under the terms of the Creative Commons Attribution License (CC BY). The use, distribution or reproduction in other forums is permitted, provided the original author(s) and the copyright owner(s) are credited and that the original publication in this journal is cited, in accordance with accepted academic practice. No use, distribution or reproduction is permitted which does not comply with these terms. 